\title{
Growth, cycles and convergence in US regional time series
}

\author{
Vasco M. Carvalho and Andrew C. Harvey \\ Faculty of Economics and Politics, Cambridge University
}

August 26, 2002

\begin{abstract}
This article reports the results of fitting unobserved components (structural) time series models to data on real income per capita in eight regions of the United States. The aim is to establish stylised facts about cycles and convergence. A new model is developed in which convergence components are combined with a common trend and cycles. These convergence components are formulated as a second-order error correction mechanism which allows temporary divergence while imposing eventual convergence. This model is able to characterise the convergence patterns of all but the two richest US regions; these appear to have been diverging from the others in recent years. The use of unit root tests for testing convergence is critically assessed in the light of these results.

KEYWORDS: Balanced growth, error correction mechanism, Kalman filter, signal extraction, unobserved components.
\end{abstract}

JEL classification: C32, O40.

\section{Introduction}

The revival of growth theory in the mid-eighties has led to a substantial empirical research effort. The analysis of regional growth dynamics has proved to be an particularly fertile ground for this literature: to the extent that common membership 
of a nation tends to assure factor mobility and to eliminate technological, preference and institutional differences, the basic assumptions of the neoclassical growth model are likely to be met, thus rendering regional data sets the ideal testbed for assessing the absolute convergence implications of the theory. And yet, much disagreement remains. The plethora of contradicting results stems mainly from the use of different econometric methods, developed or applied over the last two decades. The result is a growing dissatisfaction with the current state of growth econometrics, as set out by Brock and Durlauf (2000) and Durlauf (2001).

In view of the above dissatisfaction, Durlauf (2001, p68) calls for econometrics to 'clarify how empirical workers should elucidate data patterns and draw inferences concerning growth'. This article is a response to this challenge. We first show how fitting multivariate unobserved components (structural) time series models to data on real income per capita can help to establish stylised facts about cycles and convergence. Rather than simply using unit root tests to decide whether convergence is taking place, we explore different scenarios concerning the extent to which convergence is taking place and which regions - if any- are converging. A new model is then developed. Its principal feature is the introduction of what we call convergence components. These are combined with a common trend and then incorporated into a model with cycles and irregular components. The convergence components are formulated as a second-order error correction mechanism which allows temporary divergence while imposing eventual convergence. Fitting the model provides a description of the movements of the series in the past and enables coherent forecasts to be made for the future.

In the light of what we find, it is apparent that tests - even when applied correctly - are at best of limited value, while at worst they are completely misleading. In any case we believe a statistical description of what is happening coupled with a forecasting mechanism is of more value. This is the case in many areas of economics. For example in the context of unit root testing and purchasing power parity (PPP), Maddala and Kim (1998, p138) state: 'A more important issue is not a test of the validity of PPP but an estimate of the time it takes for deviations from the PPP to correct themselves'. 


\subsection{Contradictory evidence on US regional convergence}

A brief review of applied work on US regional growth serves not only to set the scene for our own empirical work but also to highlight the uncertainty in the literature. Looking at the results accumulated over the last two decades, one can find competing studies concluding in favour of absolute convergence, relative (conditional) convergence and divergence, depending on the approach taken.

Using cross-sectional data, Barro and Sala-i-Martin (1992) showed that a negative correlation between initial income per capita and growth was the norm for US regions. As in cross-country comparisons, they found a slow speed of convergence; see Sala-iMartin (1996, p 1326). Unlike in cross-country studies this convergence was taken to be absolute: decreasing returns to scale should bring about a tendency for equalisation of income per capita across regions so that in the long run regions only display short run fluctuations around a common trend.

The validity of inferences drawn from the cross-sectional approach was questioned Quah (1993) who showed that the 'beta convergence' of the cross-sectional studies resulted from a weighted average and a negative value correlation between initial income per capita and growth meant only that the output differences between some pairs of countries had declined over the sample. It shed no light on heterogeneities or convergence clubs. Furthermore, Evans and Karras (1996) argued that the usual cross-sectional approach was only valid under incredible conditions. Durlauf and Quah (1999) provide a recent review of these issues.

These debates have led to an increasing interest in panel data methods for testing whether convergence is taking place and measuring the speed of convergence. Allowing for unobservable region-specific heterogeneity (individual effects) this literature has produced a very different picture of the regional convergence process in the US, characterized by rapid convergence to different steady states, that is relative convergence; see Evans and Karras (1996a, 1996b) and Evans (2000). However these findings are problematic. Such high speed of convergence are difficult to rationalise, even in traditional neoclassical growth models with a narrow view of capital. Econometrically, the typical dynamic panel formulation used is known to be subject to strong upward bias in short samples, as a result of correlation between lagged dependent variables and unobserved residuals; see Durlauf and Quah (1999). Moreover, in these short panels, fluctuations at the business cycle horizon and other high frequency movements introduce further bias, thus contributing to a systematic overestimation 
of the rate of convergence; again see Durlauf and Quah (1999).

Finally, testing for unit roots in a pure time series model finds no convergence as a rule. Bernard and Durlauf (1996 p 171) argue that '...time series tests may have poor power properties when applied to data from economies in transition'. Brown, Coulson and Engle (1990) and Carlino and Mills (1993) find no convergence in time series of US regional per capita income. Carlino and Mills (1993), Loewy and Papell (1996) and Tomljanovich and Vogelsang (2001) try to reconcile these results with the aforementioned evidence for convergence by allowing for trend breaks.

\subsection{Outline}

In section 2, we review structural time series models (STMs), as implemented in the STAMP package of Koopman et al (2000), and show how they apply to series exhibiting balanced growth. Section 3 then employs these models to capture the stylised facts surrounding the movements in income per head in US regions. The slowly changing trends show the long-run movements from which we can infer any tendencies towards convergence. We differ from researchers such as Bernard and Durlauf (1996) in defining convergence in terms of the underlying trend rather than the observations.

Distinguishing trends from cyclical movements is essential to an effective study of convergence. However, the information on cycles is of considerable interest in itself. The recent paper by Carlino and Sill (2001) uses the methodology of Vahid and Engle (1993) to decompose the series on US regions into common trends and common cycles. We do not find the resulting cycles particularly plausible - for example they are rarely below zero. By contrast our cycles, which are based on an UC model and calculated by a state space smoothing algorithm, are effectively based on two sided filters rather than one-sided filters, and their movements are much closer to NBER dating of expansions and recessions.

A multivariate model of growth and convergence is developed in Section 4 and applied in Section 5. The convergence mechanism is based on an error correction model. This can be incorporated into an unobserved components (UC) model that effectively decomposes trends into a common balanced growth path component and a set of convergence components. A key feature of the model is that the error correction mechanism is extended so as to produce smooth convergence components that can 
display temporary divergence, thereby rendering the notion of a simple measure of the speed of convergence open to question. However, definition 2 of Bernard and Durlauf (1996) is satisfied in that forecast function for the difference between any pair of regions converges to a constant. Furthermore, because the cross-section is relatively small, proper account is taking of the cross-correlations across regions.

Section 6 investigates unit root tests for convergence. In doing so we distinguish carefully between the notion of economies which have converged and those which are in the process of converging. We present the results of pairwise unit root tests and discuss the extent to which they are helpful in determining which regions can be grouped together in a convergence model.

The conclusions are set out in section 7 .

\section{Structural Time Series Models and Balanced Growth}

\section{$2.1 \quad$ Univariate models}

The local linear trend model for a set of observations, $y_{t}, t=1, . ., T$, consists of stochastic trend and irregular components, that is

$$
y_{t}=\mu_{t}+\varepsilon_{t}, \quad t=1, \ldots, T
$$

The trend, $\mu_{t}$, receives shocks to both its level and slope so

$$
\begin{array}{rlrl}
\mu_{t} & =\mu_{t-1}+\beta_{t-1}+\eta_{t}, & & \eta_{t} \sim \operatorname{NID}\left(0, \sigma_{\eta}^{2}\right), \\
\beta_{t}=\beta_{t-1}+\zeta_{t}, & & \zeta_{t} \sim \operatorname{NID}\left(0, \sigma_{\zeta}^{2}\right),
\end{array}
$$

where the irregular, level and slope disturbances, $\varepsilon_{t}, \eta_{t}$ and $\zeta_{t}$, respectively, are mutually independent and the notation $\operatorname{NID}\left(0, \sigma^{2}\right)$ denotes normally and independently distributed with mean zero and variance $\sigma^{2}$. If both variances $\sigma_{\eta}^{2}$ and $\sigma_{\zeta}^{2}$ are zero, the trend is deterministic. When only $\sigma_{\zeta}^{2}$ is zero, the slope is fixed and the trend reduces to a random walk with drift, $\beta$. Allowing $\sigma_{\zeta}^{2}$ to be positive, but setting $\sigma_{\eta}^{2}$ to zero gives an integrated random walk trend, which when estimated tends to be relatively smooth. The model is often referred to as the 'smooth trend' model.

The statistical treatment of unobserved component models, as in the STAMP package of Koopman et al (2000), is based on the state space form (SSF). Once a model has been put in SSF, the Kalman filter yields estimators of the components 
based on current and past observations. Signal extraction refers to estimation of components based on all the information in the sample. Signal extraction is based on smoothing recursions which run backwards from the last observation. Predictions are made by extending the Kalman filter forward. Root mean square errors (RMSEs) can be computed for all estimators and prediction or confidence intervals constructed. The unknown variance parameters are estimated by constructing a likelihood function from the one-step ahead prediction errors, or innovations, produced by the Kalman filter and maximizing it by an iterative procedure.

Distinguishing a long-term trend and from short-term movements is important. Short-term movements may be captured by adding a serially correlated stationary component, $\psi_{t}$, to the model. Thus

$$
y_{t}=\mu_{t}+\psi_{t}+\varepsilon_{t}, \quad t=1, \ldots, T
$$

An autoregressive process is often used for $\psi_{t}$. Another possibility is the stochastic cycle

$$
\left[\begin{array}{l}
\psi_{t} \\
\psi_{t}^{*}
\end{array}\right]=\rho\left[\begin{array}{cc}
\cos \lambda_{c} & \sin \lambda_{c} \\
-\sin \lambda_{c} & \cos \lambda_{c}
\end{array}\right]\left[\begin{array}{c}
\psi_{t-1} \\
\psi_{t-1}^{*}
\end{array}\right]+\left[\begin{array}{c}
\kappa_{t} \\
\kappa_{t}^{*}
\end{array}\right], \quad t=1, \ldots, T,
$$

where $\lambda_{c}$ is frequency in radians and $\kappa_{t}$ and $\kappa_{t}^{*}$ are two mutually independent white noise disturbances with zero means and common variance $\sigma_{\kappa}^{2}$. Given the initial conditions that the vector $\left(\psi_{0}, \psi_{0}^{*}\right)^{\prime}$ has zero mean and covariance matrix $\sigma_{\psi}^{2} \mathbf{I}$, it can be shown that for $0 \leq \rho<1$, the process $\psi_{t}$ is stationary and indeterministic with zero mean, variance $\sigma_{\psi}^{2}=\sigma_{\kappa}^{2} /\left(1-\rho^{2}\right)$ and autocorrelation function

$$
\rho(\tau)=\rho^{\tau} \cos \lambda_{c} \tau, \quad \tau=0,1,2, \ldots
$$

For $0<\lambda_{c}<\pi$, the spectrum of $\psi_{t}$ displays a peak, centered around $\lambda_{c}$, which becomes sharper as $\rho$ moves closer to one; see Harvey (1989, p 60). The period corresponding to $\lambda_{c}$ is $2 \pi / \lambda_{c}$. In the limiting cases when $\lambda_{c}=0$ or $\pi, \psi_{t}$ collapses to first-order autoregressive processes with coefficients $\rho$ and minus $\rho$ respectively. More generally the reduced form is an $\operatorname{ARMA}(2,1)$ process in which the autoregressive part has complex roots. The complex root restriction can be very helpful in fitting a model, particularly if there is reason to include more than one cycle.

Imposing the smooth trend restriction, that is setting $\sigma_{\eta}^{2}$ to zero, often allows a clearer separation into trend and cycle. 


\subsection{Multivariate models}

Suppose we have $N$ time series. Define the vector $\mathbf{y}_{t}=\left(y_{1 t}, . ., y_{N t}\right)^{\prime}$ and similarly for $\boldsymbol{\mu}_{t}, \boldsymbol{\psi}_{t}$ and $\boldsymbol{\varepsilon}_{t}$. Then a multivariate UC model may be set up as

$$
\mathbf{y}_{t}=\boldsymbol{\mu}_{t}+\boldsymbol{\psi}_{t}+\boldsymbol{\varepsilon}_{t}, \quad \boldsymbol{\varepsilon}_{t} \sim N I D\left(\mathbf{0}, \boldsymbol{\Sigma}_{\varepsilon}\right), \quad t=1, \ldots, T,
$$

where $\boldsymbol{\Sigma}_{\varepsilon}$ is an $N \times N$ positive semi-definite matrix. The trend is

$$
\begin{aligned}
\boldsymbol{\mu}_{t} & =\boldsymbol{\mu}_{t-1}+\boldsymbol{\beta}_{t-1}+\boldsymbol{\eta}_{t}, \quad \boldsymbol{\eta}_{t} \sim \operatorname{NID}\left(\mathbf{0}, \boldsymbol{\Sigma}_{\eta}\right) \\
\boldsymbol{\beta}_{t} & =\boldsymbol{\beta}_{t-1}+\boldsymbol{\zeta}_{t}, \quad \boldsymbol{\zeta}_{t} \sim N I D\left(\mathbf{0}, \boldsymbol{\Sigma}_{\zeta}\right),
\end{aligned}
$$

With $\boldsymbol{\Sigma}_{\eta}=\mathbf{0}$, we get the smooth trend model. With $\boldsymbol{\Sigma}_{\zeta}=\mathbf{0}$, we get the random walk plus drift.

The similar cycle model, introduced by Harvey and Koopman (1997) is

$$
\left[\begin{array}{c}
\boldsymbol{\psi}_{t} \\
\boldsymbol{\psi}_{t}^{*}
\end{array}\right]=\left[\rho\left(\begin{array}{cc}
\cos \lambda_{c} & \sin \lambda_{c} \\
-\sin \lambda_{c} & \cos \lambda_{c}
\end{array}\right) \otimes \mathbf{I}_{N}\right]\left[\begin{array}{c}
\boldsymbol{\psi}_{t-1} \\
\boldsymbol{\psi}_{t-1}^{*}
\end{array}\right]+\left[\begin{array}{c}
\boldsymbol{\kappa}_{t} \\
\boldsymbol{\kappa}_{t}^{*}
\end{array}\right], \quad t=1, \ldots, T,
$$

where $\boldsymbol{\psi}_{t}$ and $\boldsymbol{\psi}_{t}^{*}$ are $N \times 1$ vectors and $\boldsymbol{\kappa}_{t}$ and $\boldsymbol{\kappa}_{t}^{*}$ are $N \times 1$ vectors of the disturbances such that

$$
E\left(\boldsymbol{\kappa}_{t} \boldsymbol{\kappa}_{t}^{\prime}\right)=E\left(\boldsymbol{\kappa}_{t}^{*} \boldsymbol{\kappa}_{t}^{*^{\prime}}\right)=\boldsymbol{\Sigma}_{\kappa}, \quad E\left(\boldsymbol{\kappa}_{t} \boldsymbol{\kappa}_{t}^{*^{\prime}}\right)=\mathbf{0},
$$

where $\boldsymbol{\Sigma}_{\kappa}$ is an $N \times N$ covariance matrix. The model allows the disturbances to be correlated across the series. Because the damping factor and the frequency, $\rho$ and $\lambda_{c}$, are the same in all series, the cycles in the different series have similar properties; in particular their movements are centred around the same period. This seems eminently reasonable if the cyclical movements all arise from a similar source such as an underlying business cycle. Furthermore, the restriction means that it is often easier to separate out trend and cycle movements when several series are jointly estimated.

\subsection{Stability and balanced growth}

The balanced growth UC model is a special case of (6):

$$
\mathbf{y}_{t}=\mathbf{i} \mu_{t}+\boldsymbol{\alpha}+\boldsymbol{\psi}_{t}+\varepsilon_{t}, \quad \quad t=1, \ldots, T,
$$


where $\mu_{t}$ is a univariate local linear trend as in $(2, \mathbf{i}$ is a vector of ones, and $\boldsymbol{\alpha}$ is an $N \times 1$ vector of constants. If $\mu_{t}$ is initialised with a diffuse prior, then $\boldsymbol{\alpha}$ must be subject to a constraint so it contains only $N-1$ free parameters, for example there may be one zero entry. Alternatively, $\mu_{0}$ may be set to zero. Note that although the levels may be different, the slopes are the same, irrespective of whether they are fixed or stochastic.

A balanced growth model implies that the series have a stable relationship over time. This means that there is a full rank $(N-1) \times N$ matrix, $\mathbf{D}$, with no null columns and the property that $\mathbf{D i}=\mathbf{0}$, thereby rendering $\mathbf{D} \mathbf{y}_{t}$ jointly stationary. The rows of $\mathbf{D}$ may be termed balanced growth co-integrating vectors. Typically each row will contain a one, a minus one and zeroes elsewhere. For example, one country may be used as a benchmark or numeraire. The multivariate stationarity test described in Nyblom and Harvey (2000) and Hobijn and Franses (2000) may be used to test the null hypothesis ${ }^{1}$ of balanced growth; the test statistic is invariant to the choice of $\mathbf{D}$.

If the series are stationary in first differences, balanced growth may be incorporated in a vector error correction model (VECM) by writing

$$
\Delta \mathbf{y}_{t}=\boldsymbol{\delta}+\boldsymbol{\Gamma} \mathbf{D} \mathbf{y}_{t-1}+\sum_{r=1}^{p} \boldsymbol{\Phi}_{r}^{*} \Delta \mathbf{y}_{t-r}+\boldsymbol{\xi}_{t}, \quad \operatorname{Var}\left(\boldsymbol{\xi}_{t}\right)=\boldsymbol{\Sigma}_{\xi}
$$

where the $\boldsymbol{\Phi}_{r}^{* \prime} s$ are $N \times N$ matrices, $\mathbf{D}$ is as defined in the previous paragraph and the matrix $\boldsymbol{\Gamma}$ is $N \times(N-1)$. The system has a single unit root, guaranteed by the fact that $\mathbf{D i}=\mathbf{0}$. The constants in $\boldsymbol{\delta}$ contain information on the common slope, $\beta$, and on the differences in the levels of the series, as contained in the vector $\boldsymbol{\alpha}$. These differences might be parameterised with respect to the contrasts in $\mathbf{D} \mathbf{y}_{t-1}$. For example if $\mathbf{D} \mathbf{y}_{t}$ has elements $y_{i t}-y_{i+1, t}, i=1, . ., N-1$, then $\alpha_{i}$, the $i-t h$ element of the $(N-1) \times 1$ vector $\boldsymbol{\alpha}$, is the gap between $y_{i}$ and $y_{i+1}$. In any case, $\boldsymbol{\delta}=\beta\left(\mathbf{I}-\sum_{j=1}^{p} \mathbf{\Phi}_{j}^{*}\right) \mathbf{i}-\boldsymbol{\Gamma} \boldsymbol{\alpha}$. Estimation by OLS applied to each equation in turn is fully efficient since each equation contains the same explanatory variables.

A UC balanced growth model in which the common trend is a random walk plus drift may be approximated by (11). This can be useful as a baseline for forecasting and for giving initial estimates of some parameters. However, the VECM does not

\footnotetext{
${ }^{1}$ Hobijn and Franses (2000) actually say that they are testing whether the countries "are converging". In fact stationarity tests are not useful in this context since initial values some way from the equilibrium will cause them to reject.
} 
provide the description that can be obtained by extracting unobserved components.

\subsection{Similar and common cycles}

In the similar cycle model the extent to which the cycles move together depends on the correlations between the disturbances driving them since

$$
\boldsymbol{\Sigma}_{\psi}=\left(1-\rho^{2}\right)^{-1} \boldsymbol{\Sigma}_{\kappa}
$$

Using principal components analysis we can decompose $\boldsymbol{\Sigma}_{\kappa}$ as $\mathbf{E D E} \mathbf{E}^{\prime}$ where $\mathbf{D}$ is a diagonal matrix of eigenvalues and $\mathbf{E}$ is the corresponding matrix of eigenvectors. The principal components themselves are contained in the series in the $N \times 1$ vector $\boldsymbol{\psi}_{t}^{\dagger}=\mathbf{E}^{\prime} \boldsymbol{\psi}_{t}$, while the variances of the disturbances driving the $j-t h$ principal component is $d_{j}$, the $j-t h$ diagonal element of $\mathbf{D}$. These principal component cycles can be entered into the model (6) by writing

$$
y_{t}=\boldsymbol{\mu}_{t}+\mathbf{E} \boldsymbol{\psi}_{t}^{\dagger}+\varepsilon_{t} .
$$

The proportion of the variance of the $i-t h$ cycle accounted for by the $j-t h$ principal component is $e_{i j}^{2} d_{j} / \sigma_{\psi i}^{2}$; note that $\sigma_{\psi i}^{2}=\Sigma_{j} e_{i j}^{2} d_{j}$.

We can create a set of $N$ standardised principal component cycles as $\boldsymbol{\psi}_{t}^{*}=$ $\mathbf{D}^{-1 / 2} \mathbf{E}^{\prime} \boldsymbol{\psi}_{t}$ with $\boldsymbol{\Sigma}_{\kappa^{*}}=\mathbf{I}$. The factor loadings are then $\boldsymbol{\Theta}=\mathbf{E D}^{1 / 2}$ and (12) becomes

$$
\mathbf{y}_{t}=\boldsymbol{\mu}_{t}+\Theta \psi_{t}^{*}+\varepsilon_{t}
$$

This formulation is useful as a starting point for factor rotations.

If $\boldsymbol{\Sigma}_{\psi}$ is less than full rank, there are common cycles. A model can be estimated with a given rank as described in Koopman et al (2000). If the rank of $\boldsymbol{\Sigma}_{\psi}$ is one, there is a single common cycle and the model can be written

$$
y_{i t}=\mu_{i t}+\theta_{i} \psi_{t}^{\dagger}+\varepsilon_{i t}, \quad i=1, \ldots, N
$$

where $\psi_{t}^{\dagger}$ is a scalar cycle and one of the $\theta_{i}^{\prime} s$ is set equal to unity; note that there is no need for a constant as in (10). A single common cycle is a common feature in the sense of Engle and Kozicki (1993) in that it may be removed by a linear combination, $\overline{\boldsymbol{\theta}}$, of the observations with the property that $\overline{\boldsymbol{\theta}}^{\prime} \boldsymbol{\theta}=0$, where the $N \times 1$ vector $\boldsymbol{\theta}=\left(\theta_{1}, \ldots, \theta_{N}\right)^{\prime}$. 
Testing the null hypothesis of a single common cycle is not straightforward. However, for the case of $N=2$, the distribution of the LR statistic is an even mixture of $\chi_{0}^{2}$ and $\chi_{1}^{2}$; see Harvey (1989, p236). Thus the $5 \%$ critical value is 2.71 .

The weights in (13) allow the common cycle to appear in each series with a different amplitude. If $\theta_{i}=1, i=1, . ., N$, then the cycles in each equation are said to be identical.

\section{Stylised facts: trends and cycles}

In this section we analyse trend and cyclical dynamics in the logarithms of real per capita incomes in US census regions: New England (NE), Mid East (ME), Great Lakes (GL), Plains (PL), South East (SE), South West (SW), Rocky Mountains $(\mathrm{RM})$ and Far West $(\mathrm{FW})$. The data were obtained from the Bureau of Economic Analysis and deflated by the US implicit price deflator $(1996=100)$. Figure 1 shows annual observations for the eight US census regions from 1950 to 1999. Carlino and Mills (1993) use annual data from 1929 (to 1990). However, because the fluctuations in the 1930s and 1940s are so much bigger than the cycles after 1950 it is difficult to model the whole series satisfactorily. The introduction of a trend break, as in Carlino and Mills (1993) and Loewy and Papell (1996), does not really address this problem.

Figure 2 shows quarterly seasonally adjusted observations for the same regions from 1969:1 to 1999:4. 


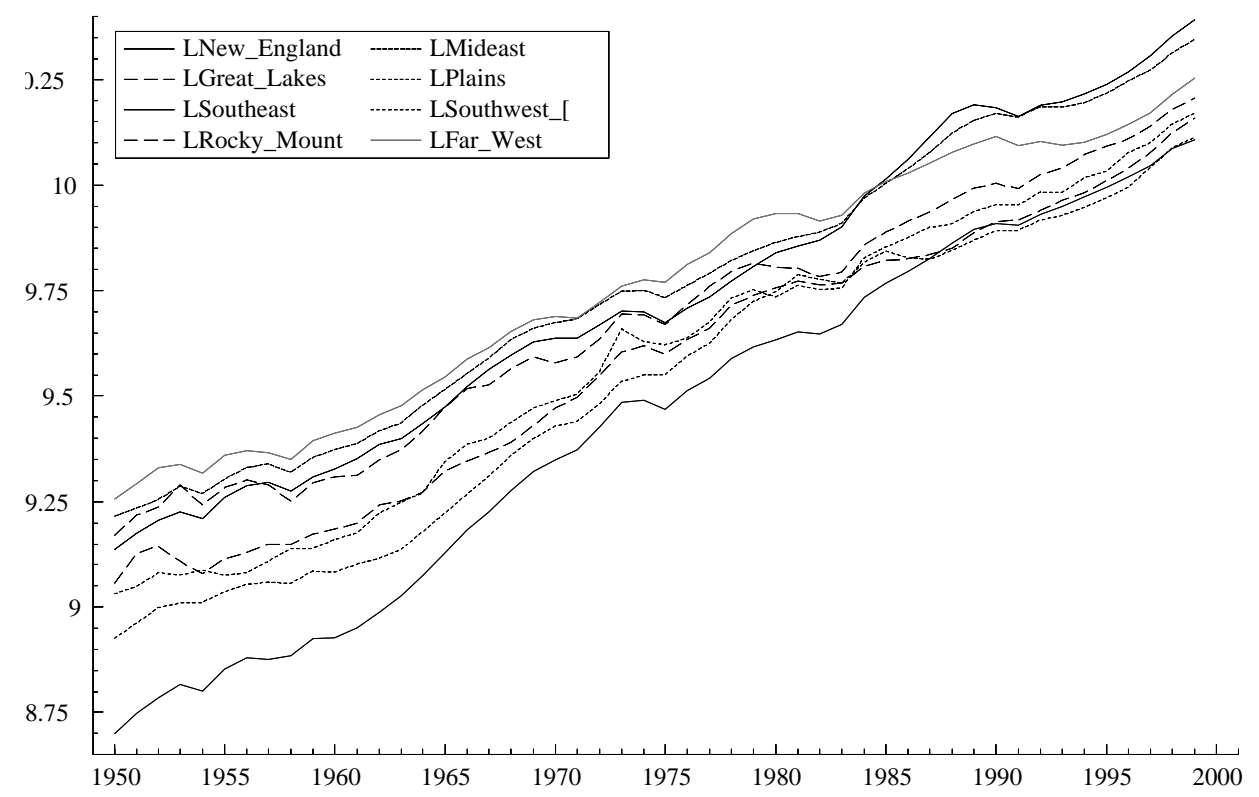

Figure 1: 8 US census regions. Annual data.

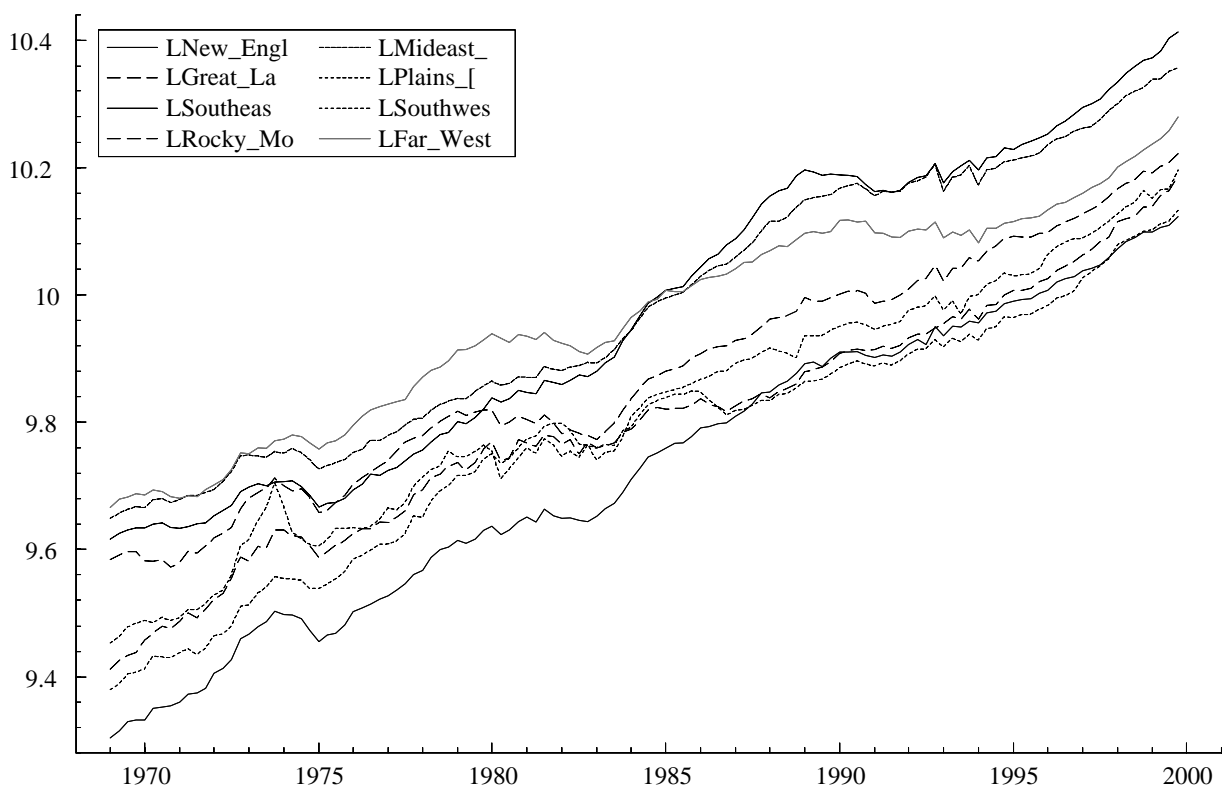

Figure 2: 8 US census regions. Quarterly data. 
We report the results of fitting unrestricted multivariate structural time series models of the form (6) with smooth trends in order to obtain some idea of stylised facts. Estimation of this and all other models below was done using program routines ${ }^{2}$ written in the OX 3.0 language (Doornik, 1999) with use being made of the SsfPack package for state space algorithms of Koopman, Shephard and Doornik (1999). Programs are available on request.

All parameters were estimated by maximum likelihood as described in Section 3 and variances reported are multiplied by $10^{7}$. The estimated covariance matrices are reported by showing the variances on the main diagonal while the entries above contain the cross-correlations. All graphs show the estimated components as extracted by the state-space smoothing algorithm.

The results presented here are primarily for the annual series. It would be tedious to repeat the corresponding results for the quarterly series and they have been put in the appendix.

\subsection{Cycles}

The smoothed cyclical components, $\widetilde{\psi}_{i t \mid T}$, for the eight annual regional series are shown in Figure 3. They track the nationwide NBER reference cycles quite accurately: the 1954, 1961, 1970, 1975, 1980, 1982 and the 1991 recessions all show up with a high degree of coherence across regions, unlike in Carlino and Sill (2001). The period of the cycle is 5.3 years with a damping factor, $\rho$, of 0.80 ; the baseline average cyclical duration referenced by NBER for the 1945-91 period is 5.1 years. Moving to quarterly frequency the main results continue to hold and are reproduced in the appendix. All recessions from 1969 onwards are evident though the 1991 recession is less marked. The period for the quarterly series is 4.25 years with a damping factor of 0.85 .

\footnotetext{
${ }^{2}$ The current version of STAMP has difficulty coping with a cross-section of size eight.
} 


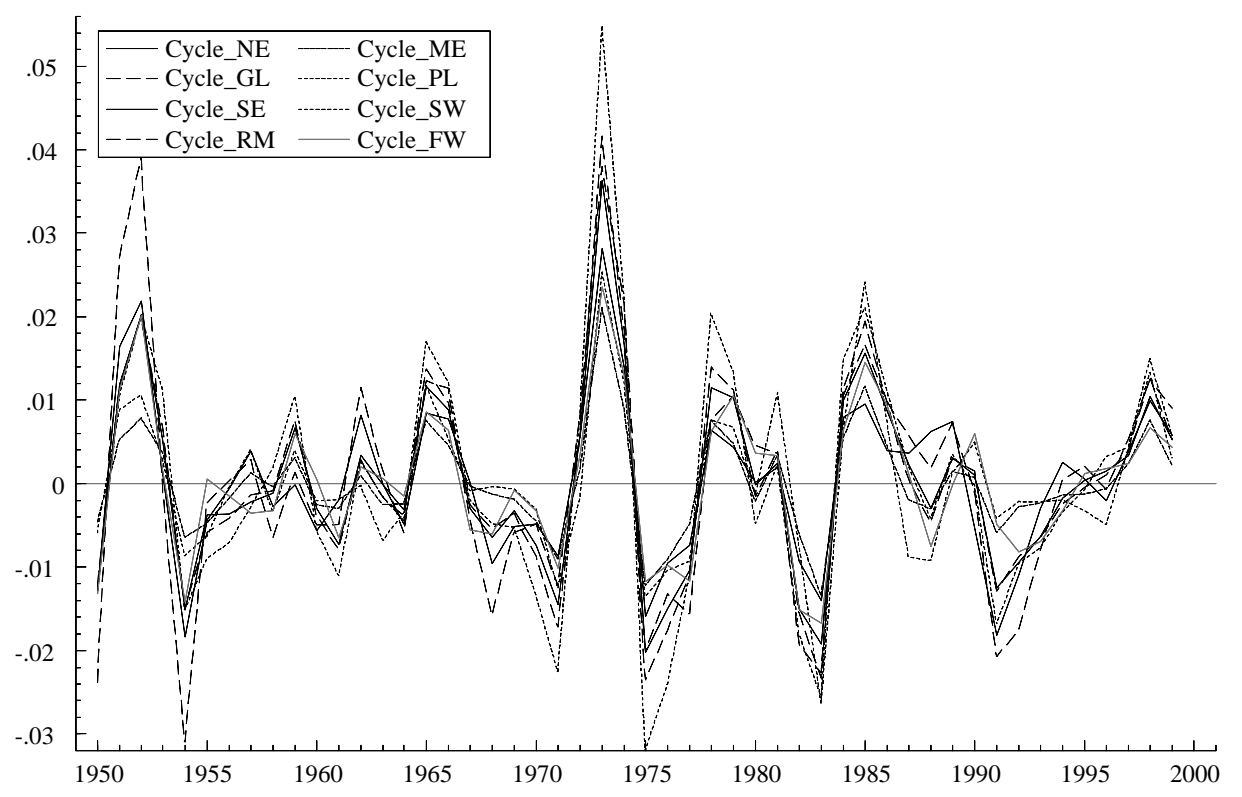

Figure 3: Cyclical components for annual data.

The estimate of the cycle disturbance covariance matrix, $\boldsymbol{\Sigma}_{\kappa}$, displays considerable differences in the volatility of regional cyclical disturbances, with the variance of cyclical disturbance in the most volatile region (PL) being almost six times as great as that of the least volatile region (ME). This heterogeneity holds also for the quarterly data. These results regarding differences in cyclical volatility are similar to those reported in Carlino and Sill (2001, 452) based on Beveridge-Nelson trends. However, our ordering of the regions in terms of volatility differs from theirs. Furthermore, we find, both for annual and quarterly data, that the richest regions (NE, ME and FW) are those with the least volatile cyclical components.

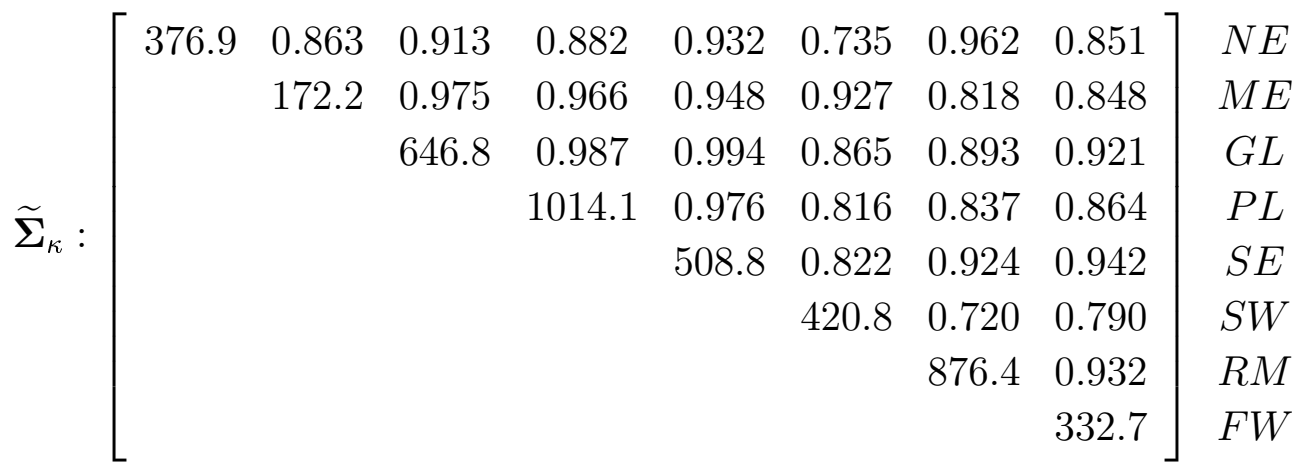


There are high, positive correlations between the cyclical disturbances in all regions. This reflects the dominance of the national cycle, an issue investigated in more detail when we look at principal components. The correlations fall somewhat when we move to the more volatile quarterly series. Thus the Plains and Great Lakes regions, two of the most volatile regions, have the lowest correlations. By contrast, the cycles in the two richest regions, New England and Mideast, are almost perfectly correlated.

The lower correlations in the quarterly data are also reflected in principal components analysis. Thus for the annual data the first principal component of $\widetilde{\boldsymbol{\Sigma}}_{\kappa}$ accounts for $91 \%$ of the total variance while the second accounts for a further $5 \%$. The corresponding figures for quarterly data are $75 \%$ and $16 \%$ respectively. Table 1 shows the weights applied to each of the first two principal component cycles to obtain the regional cycle and the percentage variance in each regional cycle contributed by the first two components; see (12) and the text below it. At the annual frequency the proportion of the first (second) cyclical component in each region is larger (smaller) than at the quarterly frequency. The second component in the annual series also has more negative weights.

Table 1a Weights assigned to the first two principal components in cycles for each series: annual data

\begin{tabular}{|c|c|c|c|c|}
\hline Region & Weight, $e_{i 1}$ & Weight, $e_{i 2}$ & Proportion, 1 & Proportion, 2 \\
\hline$N E$ & 0.29 & 0.29 & 0.89 & 0.05 \\
\hline$M E$ & 0.20 & -0.23 & 0.92 & 0.07 \\
\hline$G L$ & 0.40 & -0.18 & 0.99 & 0.01 \\
\hline$P L$ & 0.49 & -0.38 & 0.94 & 0.03 \\
\hline$S E$ & 0.36 & -0.01 & 0.99 & 0.0003 \\
\hline$S W$ & 0.28 & -0.45 & 0.73 & 0.10 \\
\hline$R M$ & 0.44 & 0.68 & 0.88 & 0.11 \\
\hline$F W$ & 0.27 & 0.17 & 0.88 & 0.02 \\
\hline & & & & \\
\hline
\end{tabular}

Table 1b Weights assigned to the first two principal components in cycles for each series: quarterly data 


\begin{tabular}{|c|c|c|c|c|}
\hline Region & Weight, $e_{i 1}$ & Weight, $e_{i 2}$ & Proportion, 1 & Proportion, 2 \\
\hline$N E$ & 0.27 & 0.25 & 0.82 & 0.15 \\
\hline$M E$ & 0.25 & 0.23 & 0.82 & 0.15 \\
\hline$G L$ & 0.38 & 0.06 & 0.79 & 0.004 \\
\hline$P L$ & 0.50 & -0.84 & 0.62 & 0.38 \\
\hline$S E$ & 0.38 & 0.19 & 0.93 & 0.05 \\
\hline$S W$ & 0.36 & 0.21 & 0.76 & 0.05 \\
\hline$R M$ & 0.36 & 0.18 & 0.83 & 0.05 \\
\hline$F W$ & 0.26 & 0.24 & 0.69 & 0.13 \\
\hline & & & & \\
\hline
\end{tabular}

The $e_{i j}^{\prime} s$ not only show the weight given to each principal component in forming the cycle for each region, but they are also the weights given to the regional cycles in constructing the principal components. Both at the annual and quarterly frequencies, the first principal component is a composite of the regional cycles in which the weights are all positive and of a similar order of magnitude. The second principal component contrasts the regional cycles. For example, for quarterly data, it takes account of the higher volatility in the Plains region whilst for the annual data it does the same for the high-volatility Rocky Mountains.

By standardising the weights, $e_{i 1}$, for the first principal component so that they sum to one, it is possible to construct a first principal component cycle ${ }^{3}$ from the individual regional cycles. For annual data this principal component is shown in figure 4 , where it is contrasted with the smoothed cyclical component series extracted from the univariate US annual series. The two cycles are very close and the same is true for quarterly data. This again illustrates the point that the main source of regional cyclical volatility is a component closely related to the national business cycle.

\footnotetext{
${ }^{3}$ Specifically, this is the first element of $\mathbf{E}^{\prime} \widetilde{\boldsymbol{\psi}}_{t \mid T}$ and so the (unstandardised) weights are as in the first column of $\mathbf{E}$ and it is this that is reproduced under the heading $e_{i 1}$.
} 


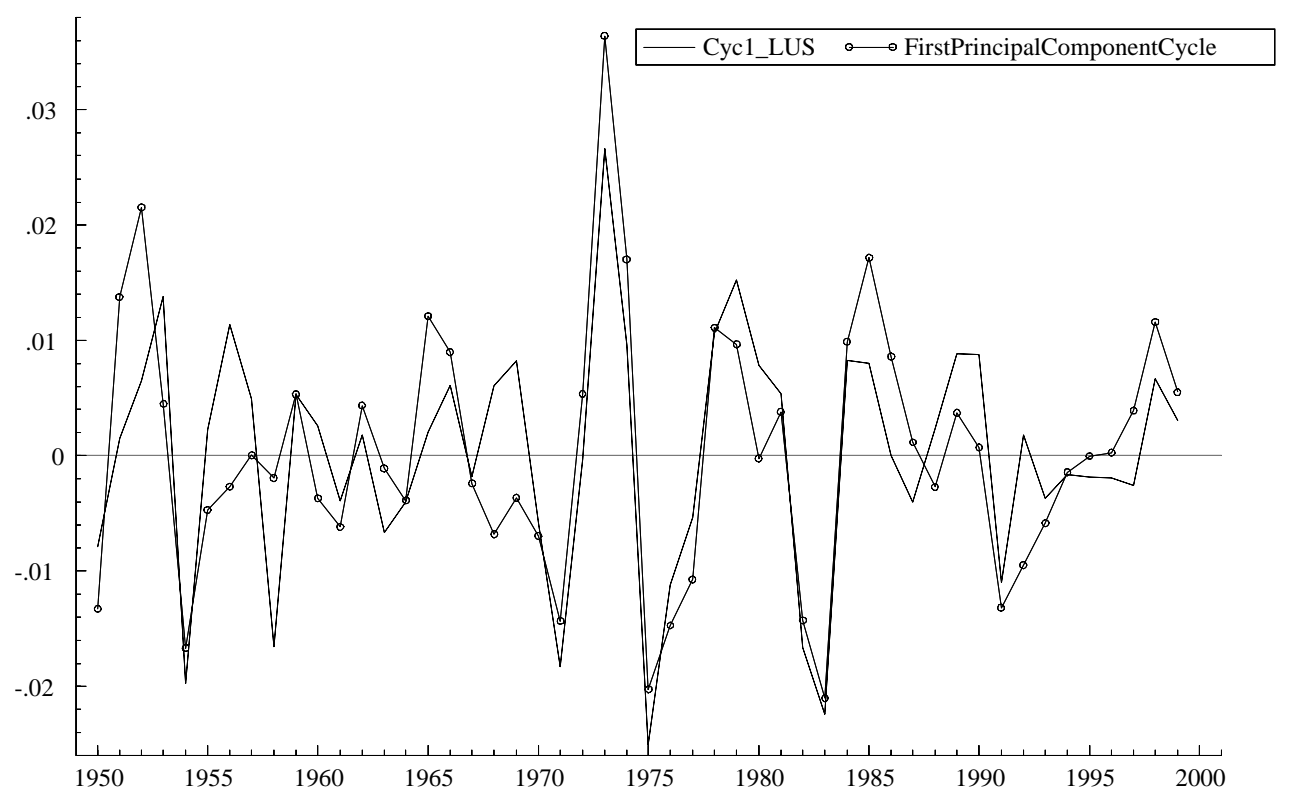

Figure 4: Cycle given by first principal component and US aggregate cycle.

Since the first principal component is so dominant we decided to see what happens when the model is estimated with a single common cycle; this is an option in STAMP. The results were not particularly useful, in that the cycle obtained bore no relation to the cycles shown in figure 4 . This is perhaps an indication that the common cycle restriction is too strong.

The other stationary component is the irregular, the covariance matrix of which is given below. It is dominated by the cycle (remember that the disturbance variance needs to be divided by $1-\rho^{2}$ to give the cycle variance), but nevertheless plays a useful role. There are no general conclusions to be drawn from the pattern of correlations. 


$$
\widetilde{\Sigma}_{\varepsilon}:\left[\begin{array}{ccccccccc}
286.2 & 0.991 & 0.877 & -0.581 & 0.824 & 0.621 & 0.425 & 0.941 \\
& 452.1 & 0.881 & -0.477 & 0.775 & 0.586 & 0.461 & 0.929 & N E \\
& & 980.9 & -0.362 & 0.768 & 0.442 & 0.205 & 0.737 & G E \\
& & & 849 & -0.536 & -0.317 & -0.340 & -0.517 & P L \\
& & & & 316.15 & 0.738 & 0.018 & 0.806 & S E \\
& & & & & 196.8 & -0.269 & 0.826 & S W \\
& & & & & & 41.3 & 0.259 \\
& & & & & & & 402.4
\end{array}\right] F W
$$

\subsection{Trends}

The smooth trends, $\widetilde{\mu}_{i t \mid T}$, extracted from the annual series by the state space smoothing algorithm are shown in figure 5. A first glance seems to indicate that crosssectional dispersion has declined, thus indicating convergence. However, closer inspection reveals otherwise. In particular, note how the trend dynamics of NE and $\mathrm{ME}$, the two wealthiest regions at the end of the sample, differ from the remaining six regions. Thus, while other relatively rich regions in the 1950's such as the Far West and Great Lakes seem to be converging to the levels of lower income regions, New England and MidEast, particularly from the late 1970's onwards, show no clear tendency to converge to some kind of common national trend. This is even more marked for the smooth trends extracted from the quarterly data. 


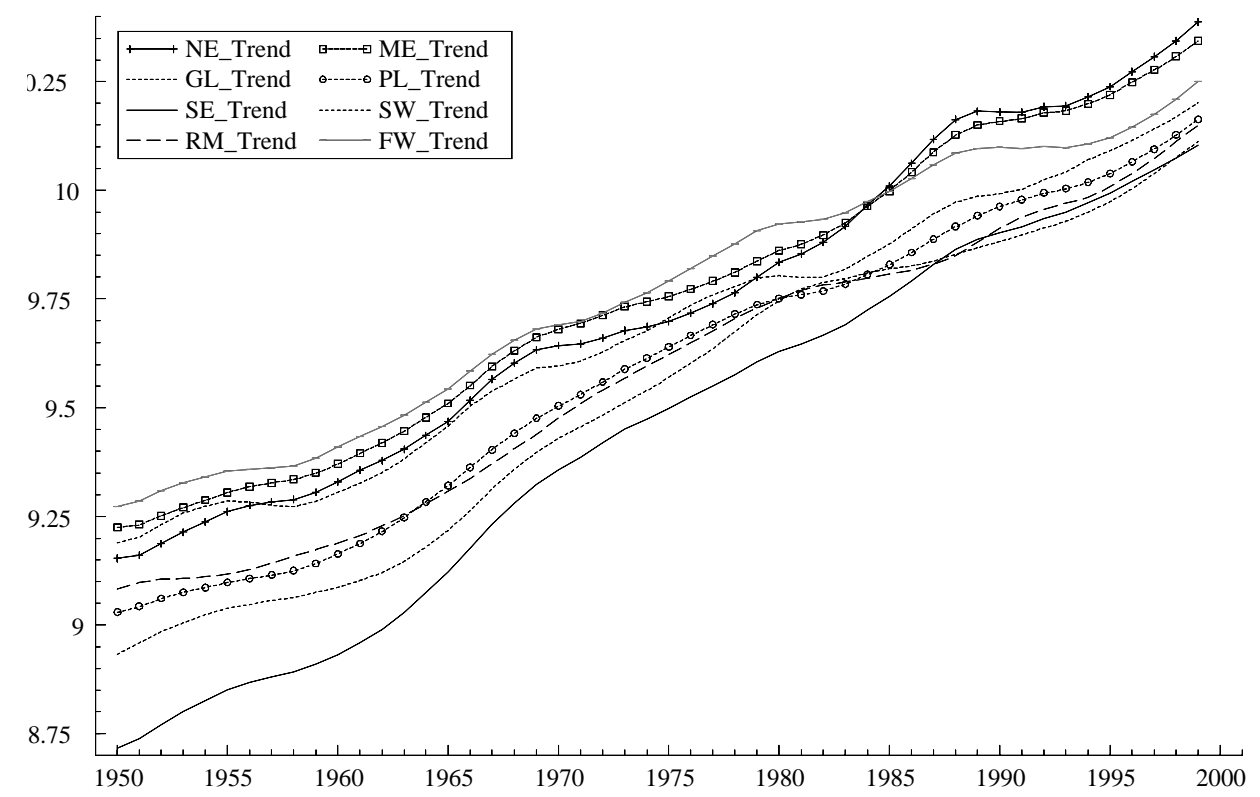

Figure 5: Smooth trends for annual data.

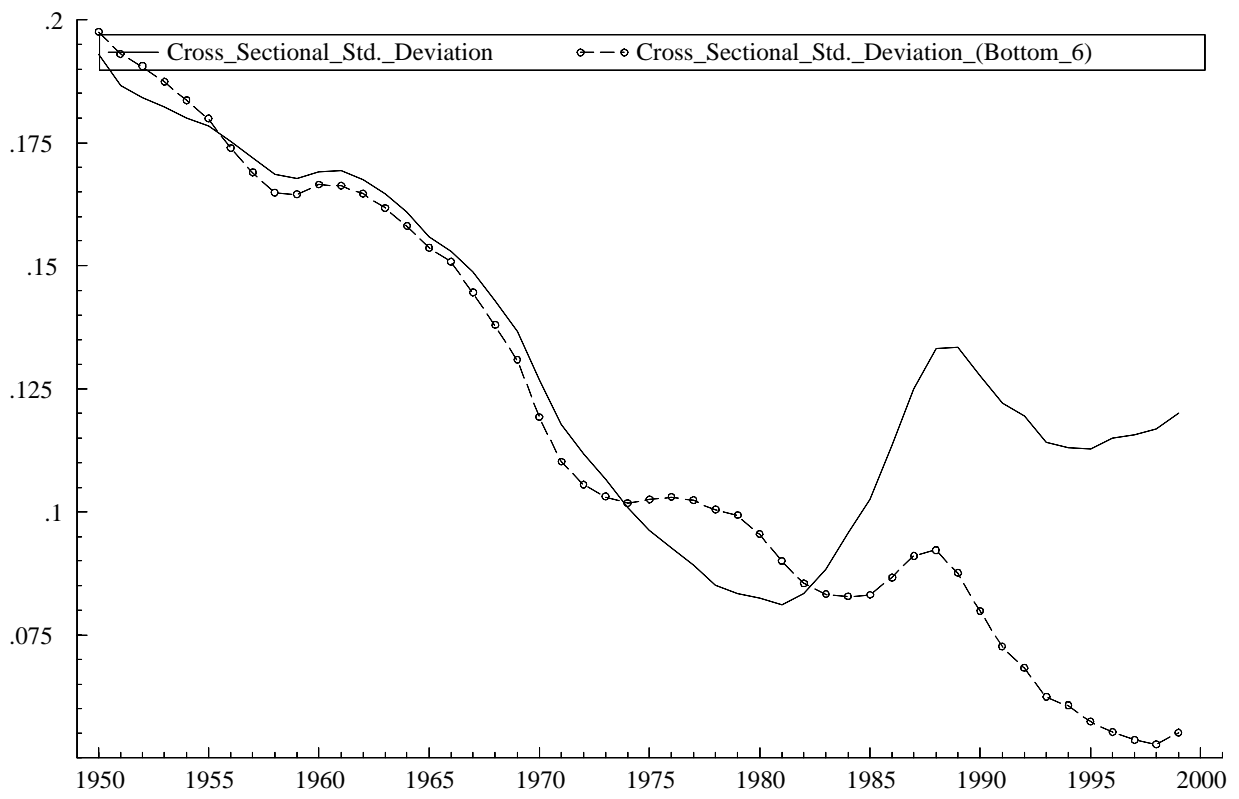

Figure 6: Cross-sectional standard deviation. 
The above conclusions are confirmed by the plot of the cross-sectional standard deviation of the smoothed trend component, $S D\left(\widetilde{\mu}_{i t \mid T}\right)$, in figure 6. Overall crosssectional dispersion, which was clearly declining until the early eighties, has since reversed its tendency so that, by the end of the sample, the situation is much the same as in the late sixties. In other words, across all regions, divergence rather than convergence seems to have been the rule since the early eighties. Excluding the two richest regions alters this conclusion. For the remaining six regions (the poorest as defined at the end of the sample) the fall in trend dispersion seems to have been continuing throughout the last two decades. Hence, there appears to be convergence of all regions apart from the two richest which are diverging from the other six regions as well as from each other.

The estimated slope disturbance covariance matrices for the annual trend component are reproduced below, where the diagonal elements are the variances of the slope disturbances and the upper triangle contains the cross-correlations. As in the cyclical component, considerable regional differences are evident in trend volatility, both at annual and quarterly frequencies. Thus in the annual data, when compared to SW, the variance of the trend component in NE is almost three times as great. This is reflected in larger fluctuations observed in the smoothed NE trend.

$$
\widetilde{\Sigma}_{\zeta}:\left[\begin{array}{ccccccccc}
1310.1 & 0.969 & 0.717 & 0.640 & 0.840 & 0.414 & -0.154 & 0.849 \\
& 665.2 & 0.716 & 0.771 & 0.891 & 0.422 & 0.005 & 0.859 & N E \\
& & 1183.7 & 0.725 & 0.822 & 0.352 & -0.189 & 0.816 & G E \\
& & & 412.8 & 0.819 & 0.569 & 0.473 & 0.861 \\
& & & & 546.5 & 0.603 & 0.068 & 0.799 & P L \\
& & & & & 579.5 & 0.554 & 0.557 \\
& & & & & & 437.8 & 0.162 \\
& & & & & & & 935.6
\end{array}\right] F W
$$

The cross-correlations between the trend disturbances show considerably more variation than those for the cycles. While the disturbances in the two wealthiest are highly correlated, some of the other disturbances actually display a negative correlation. The quarterly series show somewhat higher correlations, reflecting the fact that by the start of the series, in 1969, the series had moved more closely together. 


\section{Multivariate convergence models}

The multivariate STM has provided an idea of possible convergence patterns. We now proceed to develop a model to capture these movements. We do so by generalising of the bivariate model proposed in Harvey and Carvalho (2002).

The basic multivariate convergence model, allowing for relative convergence and a common time trend, is

$$
y_{i t}=\alpha_{i}+\beta t+\mu_{i t}, \quad i=1, \ldots, N
$$

with

$$
\mu_{i t}=\sum_{j=1}^{N} \phi_{i j} \mu_{j, t-1}+\eta_{i t}, \quad i=1, \ldots, N
$$

with $\sum_{j=1}^{N} \phi_{i j}=1$ for $i=1, \ldots, N$. This restriction can be conveniently imposed by setting $\phi_{i i}=1-\sum_{j \neq i} \phi_{i j}$. As will be shown shortly, it ensures that the system contains a unit root. As the model stands there are $N(N-1)$ parameters governing convergence. Re-formulating it as

$$
\Delta \mu_{i t}=\sum_{j \neq i} \phi_{i j}\left(\mu_{j, t-1}-\mu_{i, t-1}\right)+\eta_{i t}, \quad i=1, \ldots, N
$$

shows how the convergence of the $i-t h$ economy depends on the gap between it and each of the other $N-1$ economies. In the bivariate case, Harvey and Carvalho (2002) show that the model implies that the difference between the two economies, $y_{t}=y_{1 t}-y_{2 t}$, satisfies

$$
\Delta y_{t}=(\phi-1)\left(y_{t-1}-\alpha\right)+\eta_{t}=\delta+(\phi-1) y_{t-1}+\eta_{t}, \quad t=2, \ldots, T
$$

where $\eta_{t}=\eta_{1 t}-\eta_{2 t}, \alpha=\alpha_{1}-\alpha_{2}, \phi=1-\phi_{12}-\phi_{21}$ and $\delta=\alpha(1-\phi)$. This can be interpreted as saying that, for data in logarithms, the expected growth rate in the current period is a negative fraction of the gap between the two economies after allowing for the permanent difference, $\alpha$. Writing the model in this form accords with the notion of convergence in the cross-sectional literature, as expounded by Barro and Sala-i-Martin (1992) and others, except that there the growth rate is taken to be a linear function of the initial value, giving a model which is internally inconsistent over time; see Evans and Karras (1996, p 253). 
In matrix form

$$
\mathbf{y}_{t}=\boldsymbol{\alpha}+\beta \mathbf{i} t+\boldsymbol{\mu}_{t}
$$

with

$$
\boldsymbol{\mu}_{t}=\boldsymbol{\Phi} \boldsymbol{\mu}_{t-1}+\boldsymbol{\eta}_{t}, \quad \operatorname{Var}\left(\boldsymbol{\eta}_{t}\right)=\boldsymbol{\Sigma}_{\eta}
$$

or, in error correction form,

$$
\Delta \boldsymbol{\mu}_{t}=(\boldsymbol{\Phi}-\mathbf{I}) \boldsymbol{\mu}_{t-1}+\boldsymbol{\eta}_{t}, \quad \operatorname{Var}\left(\boldsymbol{\eta}_{t}\right)=\boldsymbol{\Sigma}_{\eta}
$$

Since each row of $\Phi$ sums to unity, $\boldsymbol{\Phi} \mathbf{i}=\mathbf{i}$. Thus setting $\lambda$ to one in $(\boldsymbol{\Phi}-\lambda \mathbf{I}) \mathbf{i}=\mathbf{0}$, shows that $\boldsymbol{\Phi}$ has an eigenvalue of one with a corresponding eigenvector consisting of ones. The other roots of $\boldsymbol{\Phi}$ are obtained by solving $|\boldsymbol{\Phi}-\lambda \mathbf{I}|=0$; they should have modulus less than one for convergence.

If we write

$$
\bar{\phi}^{\prime} \Delta \boldsymbol{\mu}_{t}=\bar{\phi}^{\prime}(\boldsymbol{\Phi}-\mathbf{I}) \boldsymbol{\mu}_{t-1}+\bar{\phi}^{\prime} \boldsymbol{\eta}_{t}
$$

it is clear that the $N \times 1$ vector of weights, $\bar{\phi}$, which gives a random walk must be such that $\overline{\boldsymbol{\phi}}^{\prime}(\boldsymbol{\Phi}-\mathbf{I})=\mathbf{0}^{\prime}$. Since the roots of $\boldsymbol{\Phi}^{\prime}$ are the same as those of $\boldsymbol{\Phi}$, it follows from writing $\left(\boldsymbol{\Phi}^{\prime}-\mathbf{I}\right) \overline{\boldsymbol{\phi}}=\mathbf{0}$ that $\overline{\boldsymbol{\phi}}$ is the eigenvector of $\boldsymbol{\Phi}^{\prime}$ corresponding to its unit root. This random walk, $\bar{\mu}_{\phi t}=\bar{\phi}^{\prime} \boldsymbol{\mu}_{t}$, is a common trend in the sense that it yields the common growth path to which all the economies converge ${ }^{4}$. The inclusion of a time trend in the model, (??), means that the overall common trend is a random walk with drift, $\beta$, and if $\boldsymbol{\alpha}$ is defined such that $\boldsymbol{\alpha}^{\prime} \overline{\boldsymbol{\phi}}=0$, each element of $\boldsymbol{\alpha}$ is a deviation from the common trend.

Unobserved components models The model in (14) may be extended so as to include cycle and irregular components. Thus

$$
y_{i t}=\alpha_{i}+\beta t+\mu_{i t}+\psi_{i t}+\varepsilon_{i t}, \quad i=1, \ldots, N
$$

In matrix terms, using the notation of (6),

$$
\mathbf{y}_{t}=\boldsymbol{\alpha}+\beta \mathbf{i} t+\boldsymbol{\mu}_{t}+\boldsymbol{\psi}_{t}+\varepsilon_{t}
$$

${ }^{4}$ This is because $\lim _{j \rightarrow \infty} \boldsymbol{\Phi}^{j}=\mathbf{i} \bar{\phi}^{\prime}$; the proof follows along the same lines as that for a well-known result on ergodic Markov chains as given, for example, in Hamilton (1994, p681). 
with $\boldsymbol{\mu}_{t}$ as in (18). The model with a smooth convergence mechanism is written as:

$$
\begin{aligned}
\mathbf{y}_{t} & =\boldsymbol{\alpha}+\boldsymbol{\mu}_{t}+\boldsymbol{\psi}_{t}+\boldsymbol{\varepsilon}_{t} \\
\boldsymbol{\mu}_{t} & =\boldsymbol{\Phi} \boldsymbol{\mu}_{t-1}+\boldsymbol{\beta}_{t-1} \\
\boldsymbol{\beta}_{t} & =\boldsymbol{\Phi} \boldsymbol{\beta}_{t-1}+\boldsymbol{\zeta}_{t}
\end{aligned}
$$

so that $\boldsymbol{\mu}_{t}$ is driven by an $N \times 1$ vector of slopes, $\boldsymbol{\beta}_{t}$, that evolve over time because of a disturbance vector, $\boldsymbol{\zeta}_{t}$, with covariance matrix, $\boldsymbol{\Sigma}_{\zeta}$. When $\boldsymbol{\Phi}=\mathbf{I}$, the $\boldsymbol{\mu}_{t}$ vector reduces to a set of smooth trend components, as in the model applied in the previous section. Harvey and Carvalho (2002) discuss the rationale behind this smooth, or second-order, convergence model and investigate its dynamic properties. As in the first-order model, (18), the forecasts converge to paths parallel to that of the common trend, $\bar{\mu}_{\phi t}$. Thus the forecast convergence condition of Bernard and Durlauf (1996, definition 2) is satisfied.

VECM Convergence may be captured by the common trend VECM of (11). The matrix $\boldsymbol{\Gamma}$ contains $N(N-1)$ free parameters and these may be estimated by OLS applied to each equation in turn. The $\boldsymbol{\Phi}$ matrix of (14) may then be estimated ${ }^{5}$ as it is given by $\boldsymbol{\Gamma D}+\mathbf{I}$. However, there is no guarantee that the estimate of $\boldsymbol{\Gamma}$ will be such $N-1$ of the roots of $\boldsymbol{\Phi}$ have modulus less than one. If the vector of gaps, $\boldsymbol{\alpha}$, is parameterised with respect to the contrasts in $\mathbf{D y}_{t-1}$, then $\boldsymbol{\delta}=\beta\left(\mathbf{I}-\sum_{j=1}^{p} \boldsymbol{\Phi}_{j}^{*}\right) \mathbf{i}-\mathbf{\Gamma} \boldsymbol{\alpha}$.

\subsection{Deviation and benchmark restrictions}

As it stands the UC model is difficult to estimate because $\boldsymbol{\Phi}$ contains $N(N-1)$ parameters. One way to impose restrictions is to specify a model in terms of deviations from a weighted average. This happens naturally if deviations (of per capita income) in regions from a national average are to be considered. Typically some regions will be bigger than others and so will receive more weight in constructing the average. However, in a more general situation we might consider the weights as giving some indication of influence. Let

$$
\bar{\mu}_{w t}=\sum_{i=1}^{N} w_{i} \mu_{i t}, \quad \sum_{i=1}^{N} w_{i}=1
$$

\footnotetext{
${ }^{5}$ We can also directly adopt the parameterisation implicit in the $\boldsymbol{\Phi}$ matrix. Although this implies a different set of explanatory variables in each equation, all satisfy the co-integrating constraints and so OLS is efficient for each equation in turn.
} 
and set

$$
\phi_{i i}=\pi_{i}+1-\pi_{i} w_{i}=\pi_{i}\left(1-w_{i}\right)+1 \quad \text { and } \quad \phi_{i j}=-\pi_{i} w_{j}, \quad i \neq j .
$$

Substituting in (15) yields

$$
\begin{aligned}
\Delta \mu_{i t} & =\pi_{i} \sum_{j \neq i} w_{j}\left(\mu_{i, t-1}-\mu_{j, t-1}\right)+\eta_{i t} \\
& =\pi_{i}\left(\mu_{i, t-1}-\bar{\mu}_{w, t-1}\right)+\eta_{i t}, \quad i=1, \ldots, N .
\end{aligned}
$$

Thus $\Delta \mu_{i t}$ depends on the gap between its own level and that of the weighted average. If $\pi_{i}=0$, then $\mu_{i t}$ is a random walk.

If $\bar{\mu}_{w, t}$ is to be a random walk, then the weights must be such that $\mathbf{w}^{\prime} \mathbf{\Phi}=\mathbf{w}^{\prime}$, that is $\sum_{i} \phi_{i j} w_{i}=w_{j}, j=1, . ., N$. The weights will only satisfy this condition if, for $i=1, . ., N, \pi_{i}=\pi$ for $w_{i} \neq 0$. This being the case we have $\mathbf{w}=\bar{\phi}$.

In the homogeneous model, when all weights are non-zero and $\pi_{i}=\pi$ for $i=$ $1, . ., N$, we are able to express the model in deviation form,

$$
\Delta\left(\mu_{i t}-\bar{\mu}_{\phi, t}\right)=\pi\left(\mu_{i, t-1}-\bar{\mu}_{\phi, t-1}\right)+\eta_{i t}-\bar{\eta}_{\phi t}, \quad i=1, \ldots, N,
$$

and any $N-1$ of these equations may be combined with the equation for $\bar{\mu}_{\phi, t}$ to give a complete system. The stability condition ${ }^{6}$ is $-2<\pi<0$.

There are a number of ways to proceed. If both $\pi_{i}^{\prime} s$ and $w_{j}^{\prime} s$ are treated as parameters, the model has $2 N-1$ parameters for $N>2$. For moderate size $N$ this parameterisation is relatively parsimonious. However, it is more appealing to focus attention on either the $\pi_{i}^{\prime} s$ or the $w_{j}^{\prime} s$. If we let the $\pi_{i}^{\prime} s$ be the same, we can estimate the $w_{j}^{\prime} s$ as $\bar{\phi}_{j}^{\prime} s$. Including $\pi$, this makes $N$ free parameters in all. The convergence process is therefore parameterised as $\phi_{i j}=-\pi \bar{\phi}_{j}, i \neq j$, and $\phi_{i i}=\pi+1-\pi \bar{\phi}_{i}$. Alternatively, we may decide to pre-assign values to the $w_{j}^{\prime} s$ and estimate the $N \pi_{i}^{\prime} s$. For the case $N=2$, these two options are equivalent. When the $\pi_{i}^{\prime} s$ are different, we can always calculate the implied weights, $\bar{\phi}_{i}$, for the common trend.

The two approaches are mixed if we set $w_{i}=0$ for some $i^{\prime} s$, and let the corresponding $\pi_{i}^{\prime} s$ be free. If $n$ are set to zero, we then, for $n<N-1$, have $N-n-1$ free

\footnotetext{
${ }^{6}$ The matrix iw' $^{\prime}$ is idempotent (though not symmetric) as its rows are identical and sum to one. Since its trace is one, it has one root of unity, while the rest are zero. The matrix $\mathbf{\Phi}=(\mathbf{1}+\pi) \mathbf{I}-\pi \mathbf{i w}^{\prime}$ also has a single unit root while the rest are $1+\pi$.
} 
$w_{i}^{\prime} s$ to estimate, together with $n \pi_{i}^{\prime} s$ and one $\pi$. When $n=N-1$, the benchmark model is obtained. In these cases, $w_{j}=\bar{\phi}_{j}$ and the $\mu_{i t}^{\prime} s$ may be put in deviation form.

Deviations from the mean If we set $w_{i}=1 / N$, then $\bar{y}_{w, t}$ is the simple mean. The implied weights may be found from the $\pi_{i}^{\prime} s$ since, provided $\pi_{j}<0$ for all $j$, $\bar{\phi}_{i}=\left(1 / \pi_{i}\right) / \sum_{j}\left(1 / \pi_{j}\right)$ as is easily seen ${ }^{7}$ from $(24)$. If we regard it reasonable to have $0 \leq \bar{\phi}_{i} \leq 1$, then the $\pi_{i}^{\prime} s$ must be less than or equal to zero. If a $\pi_{i}=0$, then we get a benchmark model, as $\bar{\phi}_{i i} \rightarrow 1$ as $\pi_{i} \rightarrow 0$. Within the context of (24) a test of $\pi_{i}=0$ can be based on standard distribution theory as $\pi_{i}=0$ does not, in itself, imply a unit root.

Benchmark model Take (without loss of generality) the Nth country as the benchmark to which all the countries converge. Then

$$
\begin{aligned}
\Delta \mu_{i t} & =\pi_{i}\left(\mu_{i, t-1}-\mu_{N, t-1}\right)+\eta_{i t}, \quad i=1, \ldots, N-1, \\
\Delta \mu_{N t} & =\eta_{N t},
\end{aligned}
$$

where the roots of the transition matrix are one and $\pi_{i}+1, i=1, . ., N-1$ so $-2<$ $\pi_{i}<0, i=1, . ., N-1$ for convergence. Note that this model is a special case of (24) obtained by setting all the weights apart from $w_{N}$ equal to zero. Since $\mu_{N t}$ is a random walk, we have

$$
\Delta\left(\mu_{i t}-\mu_{N, t}\right)=\pi_{i}\left(\mu_{i, t-1}-\mu_{N, t-1}\right)+\eta_{i t}-\eta_{N t}, \quad i=1, \ldots, N-1 .
$$

A further complication with the deviation model is that if logarithms have been taken to get the $y_{i t}^{\prime} s$, then $\bar{y}_{w, t}$ will not be the same as the the logarithm of the weighted sum of the original observations. Working in logarithms has no implications for the benchmark model.

\footnotetext{
${ }^{7}$ In matrix terms, $\boldsymbol{\Phi}=\mathbf{I}+\boldsymbol{\Pi}_{D}-\boldsymbol{\pi} \mathbf{i}^{\prime}$, where $\boldsymbol{\Pi}_{D}$ is a diagonal matrix with the elements of $\boldsymbol{\pi}$ on its diagonal. We want to find $\bar{\phi}$ such that $\boldsymbol{\Phi}^{\prime} \bar{\phi}=\left(\mathbf{I}+\boldsymbol{\Pi}_{D}-\mathbf{i} \boldsymbol{\pi}^{\prime}\right) \bar{\phi}=\bar{\phi}$. This can be done by making the $i-t h$ element of $\bar{\phi}$ proportional to the inverse of the $i-t h$ element of $\boldsymbol{\pi}$. We need to standardise so that the elements sum to one.
} 


\subsection{Autoregressive models ${ }^{8}$}

The deviation and benchmark constraints on (??) can be incorporated into an autoregressive model because $y_{i, t-1}-\bar{y}_{w, t-1}, i=1, . ., N$ are all co-integrating vectors. If one is dropped it can be reconstructed as a linear combination of the others. Thus the $\boldsymbol{\Gamma}$ and $\mathbf{D}$ matrices in (11) can be formed with suitable constraints. However, it is more convenient to set up the model as

$$
\Delta y_{i t}=\delta_{i}+\pi_{i}\left(y_{i, t-1}-\bar{y}_{w, t-1}\right)+\sum_{j=1}^{N} \sum_{r=1}^{p} \phi_{i j r}^{*} \Delta y_{j, t-r}+\eta_{i t}, \quad i=1, \ldots, N
$$

The parameters may be efficiently ${ }^{9}$ estimated by SURE, although little is likely to be lost from simply doing OLS and this may be preferable if $N$ is large. From the estimates of the $\hat{\delta}_{i}^{\prime} s$ we can solve to get $\beta$ and a set of $\alpha_{i}^{\prime} s$ for relative convergence since

$$
\delta_{i}=\beta\left[1-\sum_{j=1}^{N} \sum_{r=1}^{p} \phi_{i j}^{*}\right]-\pi_{i} \alpha_{i}, \quad i=1, \ldots, N .
$$

In a benchmark model with $\pi_{N}=0$, there are $N-1$ gaps represented by $\alpha_{i}, i=$ $1, \ldots, N-1$. More generally if we want them to be in terms of deviations from the level of the common trend, they must satisfy $\sum_{i} \bar{\phi}_{i} \alpha_{i}=0$. Recall that with a simple mean, $\bar{\phi}_{i}$ is proportional to $1 / \pi_{i}$, if all $\pi_{i}^{\prime} s$ are negative, so that the equation $\sum_{i} \alpha_{i} / \pi_{i}=0$ can be added to those in (28).

As $N$ becomes large, the above AR model runs into difficulties because of the potentially large number of $\phi_{i j r}^{*}$ parameters, $N^{2} p$ in all. It may well be the case that little explanatory power is lost by only including lagged differences of $y_{i t}$.

\subsection{Unobserved components model}

In the autoregressive framework, the natural way to proceed when the restrictions in (23) are imposed is to estimate $\pi_{i}^{\prime} s$ for a given set of pre-assigned $w_{i}^{\prime} s$. An unobserved

\footnotetext{
${ }^{8}$ The main thrust of the article concerns the UC model of the next sub-section. This section is included for completeness and for the link with unit root tests. It can be omitted without a loss of continuity.

${ }^{9}$ If the general model, (11), can be estimated, a LR test of the constraints implied by (27) can be carried out.
} 
components formulation, however, requires nonlinear optimisation with respect to the elements of $\Phi$ (as well as the other parameters, such as variances of disturbances). Since it is unclear what constraints should be imposed on the $\pi_{i}^{\prime} s$, it is relatively more attractive to assume that $\pi_{i}=\pi$ and to estimate the $\bar{\phi}_{i}$ parameters, constraining them to lie between zero and one and to sum to one. This is achieved by maximising the log-likelihood with respect to $N$ unconstrained parameters, $a_{i}$, which are linked to the $\bar{\phi}_{i}^{\prime} s$ by the equations $\bar{\phi}_{i}=a_{i}^{2} / \sum a_{i}^{2}, i=1, . ., N$. The statistical treatment of the model is based on the state space form, with the trend components being initialised with a diffuse prior; see Harvey and Carvalho (2002) for further discussion.

The model can be rewritten so as to consist of convergence components, $\mu_{i t}^{\dagger}, i=$ $1, \ldots, N$, which are deviations from the common trend, $\bar{\mu}_{\phi, t}$. Then

$$
y_{i t}=\alpha_{i}+\bar{\mu}_{\phi, t}+\mu_{i t}^{\dagger}+\psi_{i t}+\varepsilon_{i t}, \quad i=1, \ldots, N,
$$

where $\mu_{i t}^{\dagger}=\mu_{i t}-\bar{\mu}_{\phi, t}$, with

$$
\mu_{i t}^{\dagger}=\phi \mu_{i, t-1}^{\dagger}+\eta_{i t}^{\dagger}, \quad i=1, \ldots, N-1, \quad|\phi|<1
$$

where $\phi=1+\pi$, the initial conditions are $\mu_{i 0}^{\dagger}, i=1, . ., N-1$, are fixed, and

$$
\bar{\mu}_{\phi t}=\bar{\mu}_{\phi t-1}+\beta+\bar{\eta}_{\phi t}
$$

If the model is to be estimated in this form then one of the convergence equations in (30) must be dropped and the corresponding component constructed from the others. For example if $N-t h$ economy is omitted, then

$$
\mu_{N t}^{\dagger}=-\bar{\phi}_{N}^{-1} \sum_{i=1}^{N-1} \bar{\phi}_{i} \mu_{i t}^{\dagger} \quad \text { with } \quad \bar{\phi}_{N}=1-\sum_{i=1}^{N-1} \bar{\phi}_{i} .
$$

Similarly $\alpha_{N}=-\bar{\phi}_{N}^{-1} \sum_{i=1}^{N-1} \bar{\phi}_{i} \alpha_{i}$. These expressions can be substituted directly into the measurement equation for $y_{N t}$ in (29) above.

The extension to the smooth convergence processes is straightforward: the equations in (30) are replaced by

$$
\begin{aligned}
& \mu_{i t}^{\dagger}=\phi \mu_{i, t-1}^{\dagger}+\beta_{i, t-1}^{\dagger}, \quad i=1, \ldots, N-1, \quad|\phi|<1 \\
& \beta_{i, t}^{\dagger}=\phi \beta_{i, t-1}^{\dagger}+\zeta_{i t}^{\dagger}
\end{aligned}
$$


Results will be reported in terms of $\pi=\phi-1$ as in the first-order case.

If, say, $\bar{\phi}_{N}=1$, then the $N-t h$ series is a benchmark and $\bar{\mu}_{\phi t}$ is replaced by $\mu_{N t}$; in this case we can have different $\phi_{i}^{\prime} s$ in (30) and (31).

\section{Convergence and divergence in US regions}

The preliminary investigation of stylised facts reported in section 4, indicates that the two richest regions, $\mathrm{NE}$ and $\mathrm{ME}$, follow growth paths which, especially for the last two decades, seem to be diverging from the growth paths of the other regions. Hence we only attempt to fit a convergence model to the six poorer regions. The results reported are for the homogeneous model, (29), with smooth convergence as in (31) and absolute convergence, that is $\alpha_{i}=0, i=1, \ldots, N$.

For the annual data, the similar cycle parameters are estimated to be $\rho=0.79$ and $2 \pi / \lambda=8.0$ years. The distubance covariance matrix is

$\boldsymbol{\Sigma}_{\kappa}:\left[\begin{array}{cccccc|c}1795.3 & 0.867 & 0.976 & 0.877 & 0.553 & 0.914 \\ & 1373.7 & 0.938 & 0.763 & 0.854 & 0.842 & G L \\ & & 1163.4 & 0.914 & 0.720 & 0.956 & S E \\ & & & 1166.5 & 0.662 & 0.990 & S W \\ & & & & 1296.5 & 0.721 & R M \\ & & & & & 872.8\end{array}\right]$ FW

while the irregular is

$\boldsymbol{\Sigma}_{\varepsilon}:\left[\begin{array}{cccccc}834.5 & -0.357 & 0.716 & 0.534 & 0.810 & 0.675 \\ & 774.7 & -0.706 & -0.518 & -0.433 & -0.515 \\ & & 215.1 & 0.773 & 0.787 & 0.821 \\ & & & 0.5 & 0.918 & 0.984 \\ & & & & 138.9 & 0.972 \\ & & & & & 308.1\end{array}\right]$ FW

The common trend weights, $\bar{\phi}_{i}$, and convergence parameter were estimated as

\begin{tabular}{|c|c|c|c|c|c|c|}
\hline $\bar{\phi}_{G L}$ & $\bar{\phi}_{P L}$ & $\bar{\phi}_{S E}$ & $\bar{\phi}_{S W}$ & $\bar{\phi}_{R M}$ & $\bar{\phi}_{F W}$ & $\pi$ \\
\hline 0.640 & 0.298 & 0.006 & 0.0004 & 0.056 & 0.00003 & -0.1106 \\
\hline
\end{tabular}

with the covariance matrix of the disturbances being 


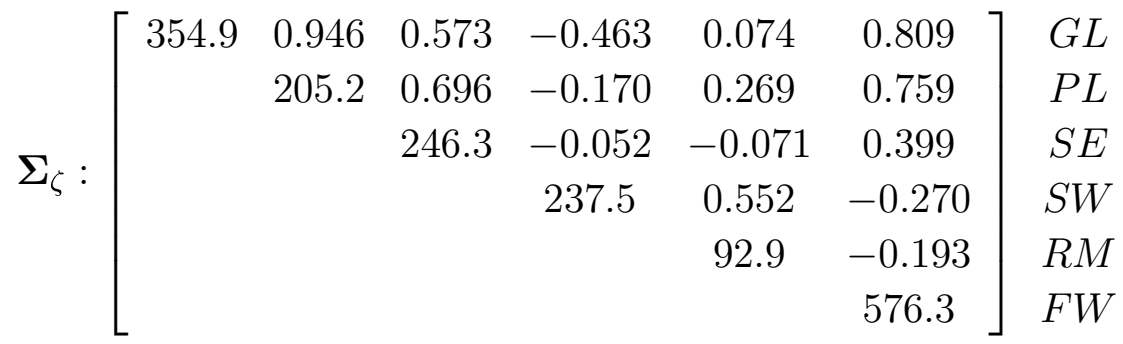

Recall that this specification not only allows us to separate trends and cycles but also separates out the long-run balanced growth path from the transitional (converging) regional dynamics, thus permitting a characterisation of convergence stylised facts. We will focus on this feature of the model, since the cycle is of secondary interest here and there is little information beyond what was presented in section 4 . Thus, figure 7 shows the smoothed estimates of the convergence components, $\mu_{i, t}^{\dagger}$, for the six regions, while figure 8 displays estimated common trend, $\bar{\mu}_{\phi, t}$, together with the estimated trends for each region.

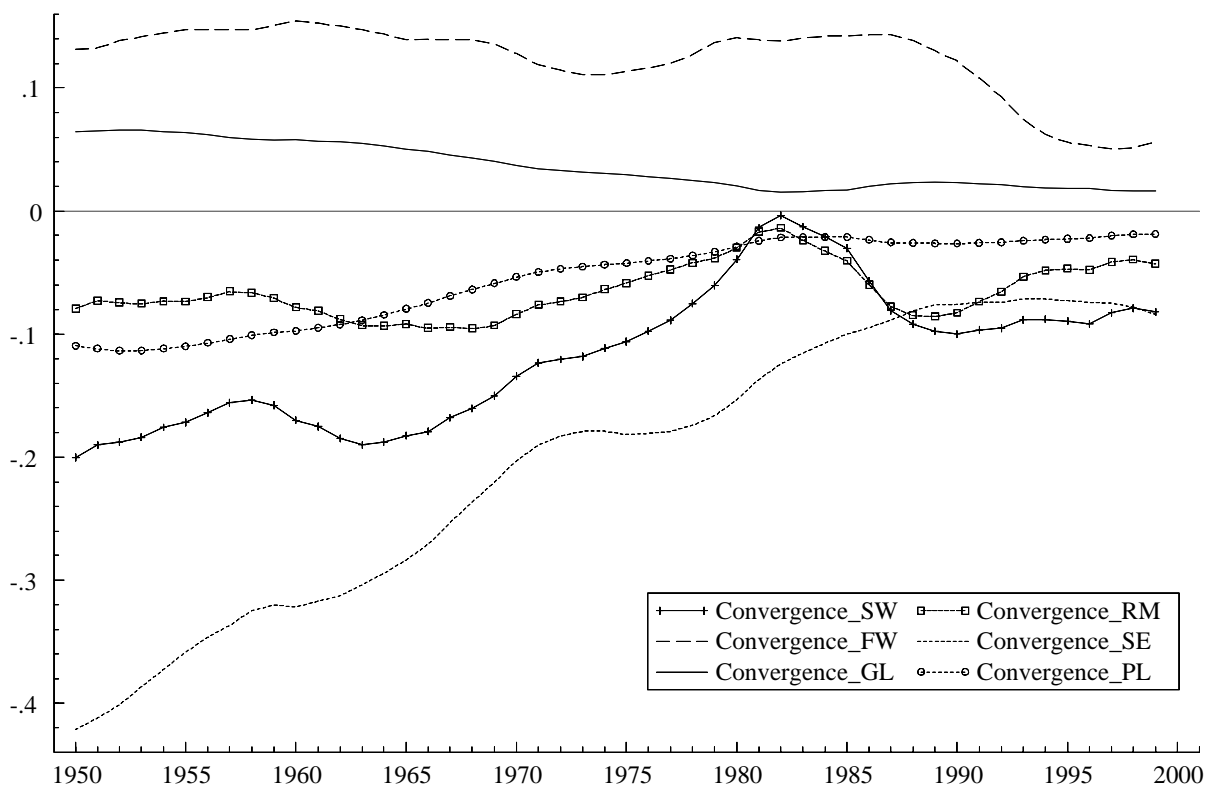

Figure 7: Convergence component for six poorest regions. 


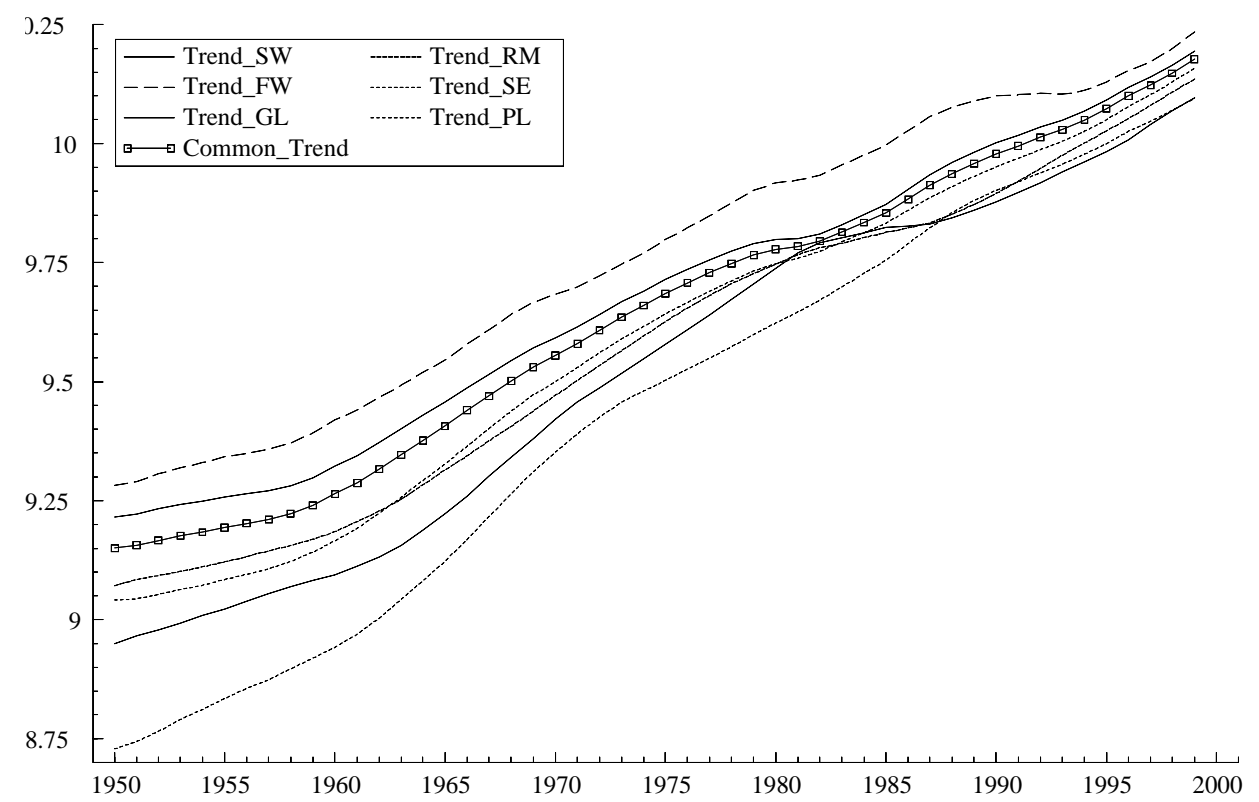

Figure 8: Common trend and growth paths for six regions group.

The negative sign on the estimate of $\pi$ is consistent with the convergence conjecture for this group of regions advanced earlier. Figures 7 and 8 provide visual support, with all six regions significantly narrowing their gap towards the common balanced growth path. The plot of the cross-sectional standard deviation computed from the smoothed trends is similar to that shown in figure 6 .

Estimation of the quarterly data set also confirms these results; see the Appendix. For both annual and quarterly data the convergence process is such that the richer and poorer regions approach a common trend which is somewhere in the middle. In fact it is basically constructed by weighting Great Lakes two-thirds and Plains one third. This suggests that we could proceed further by constructing a benchmark from the Great Lakes and Plains, thereby allowing the assumption of a single rate of convergence to be relaxed ${ }^{10}$; see the discussion in the middle of sub-section 4.1.

Note however that that beneath this convergence finding, substantial heterogeneity is evident. The convergence processes depicted $\left(\mu_{i t}^{\dagger}\right)$, are neither monotonic across time - there is no constant tendency for regional incomes disparities to decrease

\footnotetext{
${ }^{10}$ To be more specific we could set $\bar{\phi}_{i}$ to zero for all regions -apart from GL and PL- and let the corresponding $\phi_{i}$ be specific to each region. GL and PL have the same $\phi_{i}$.
} 
throughout the sample - nor homogeneous across regions. Thus while Great Lakes and Plains display smooth dynamics with most of the convergence towards the common trend taking place from 1950 to 1980, Rocky Mountains and South West display stronger convergence dynamics but only from the mid sixties onwards. Moreover, for the latter group, the 1980's are actually a period of strong divergence which is only reversed in the last decade of the sample. This type of process is also evident in the Far West region where, following a period of (slow) convergence up until the early seventies, diverging dynamics dominate from the mid seventies up until the late eighties when convergence (now stronger) resumes. Finally, the high average rate of growth of the South East region (2.83\% over the full period) translates into the strong catching-up process displayed up until the late eighties. However, during the nineties this process appears to have slowed down, or even reversed.

The estimation of the convergence model leads to slight changes in the analysis of trend variability and cross-regional correlations. The trend volatility indicated by $\widetilde{\boldsymbol{\Sigma}}_{\zeta}$ is now lower for all regions when compared to the (same regions of the) baseline smooth trend model, while there are more negative cross-correlations.

Multi-step forecasts enable us to analyse further the convergence dynamics implied by the estimates. Figure 9 shows the forecasts of the convergence components for the six regional series over a twenty year horizon (2000-2019), while figure 10 contrasts the different paths. 


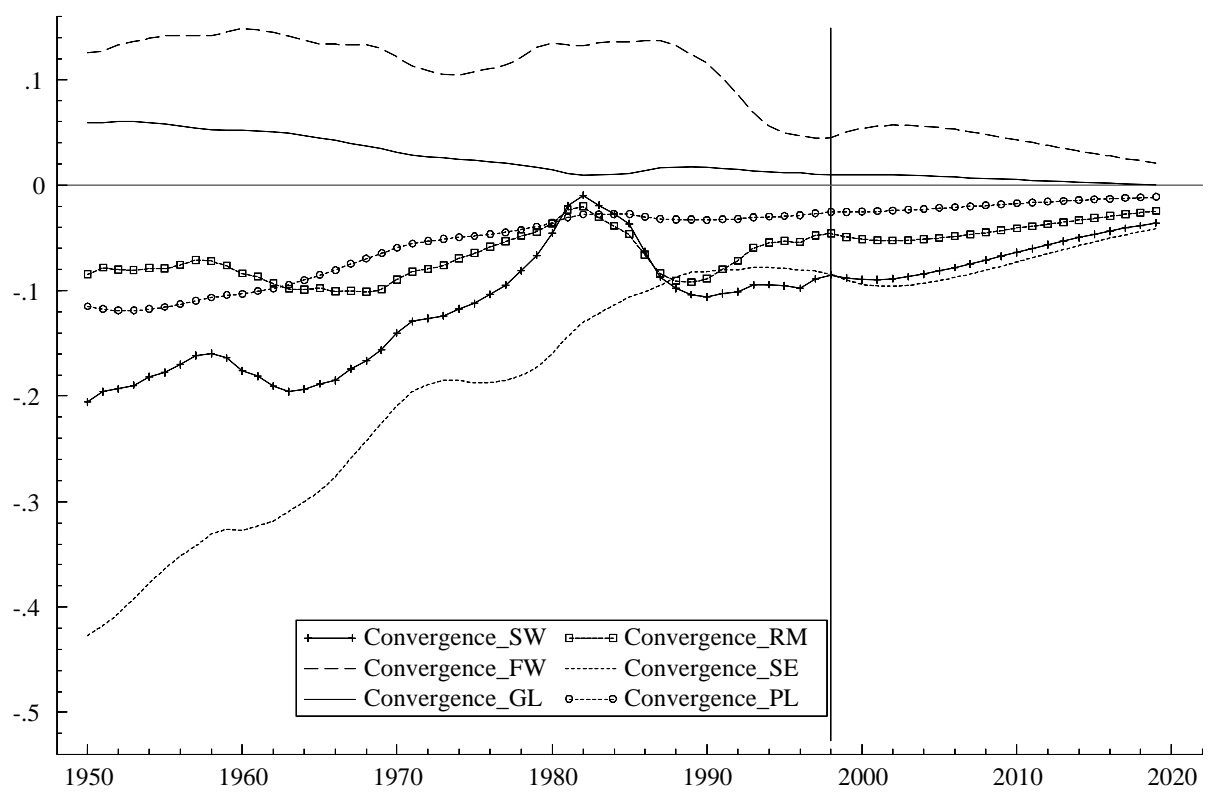

Figure 9: Forecasts for convergence component.

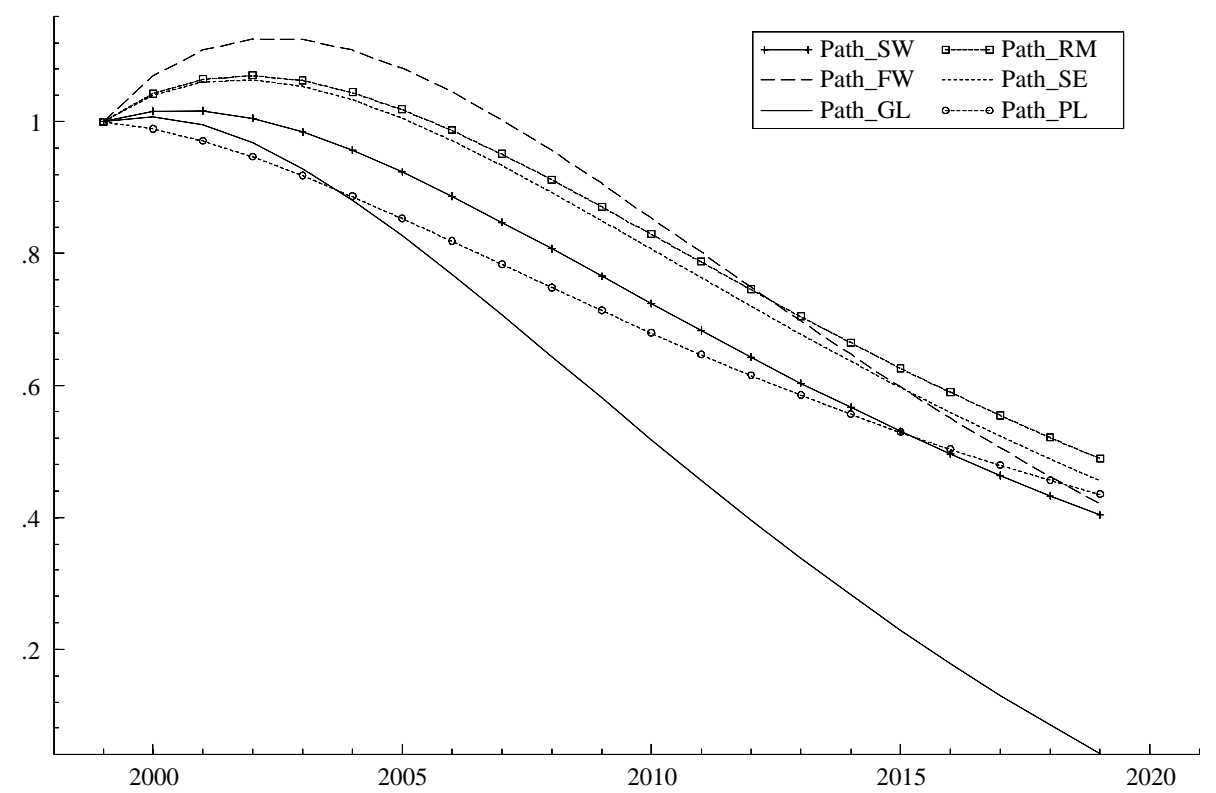

Figure 10: Forecast path $(1999=1)$. 
The striking feature of figures 9 and 10 is not the eventual convergence, but rather the prediction of divergence in the short run. Thus, although Plains and Great Lakes converge rapidly to the growth path of the common trend, which is hardly surprising given the composition of the common trend, the Far West, Rocky Mountains, South East and South West are all expected to widen their income gap, relative to the common trend, during the first five years of the forecast period. Only then do they resume their convergence towards the common trend and even then with noticeable differences in dynamics. Thus, by 2019 (2009), while the Great Lakes are expected to have removed $95 \%$ (50\%) of the 1999 gap, the remaining six regions will have removed only between $51 \%$ (17\%) -for the SW - and 60\% (28\%) - for RM- of their respective gaps.

This temporary divergence is a feature of the smooth convergence model. As Harvey and Carvalho (2002) show, the second-order error correction specification not only admits slower changes but also, when the convergence process stalls, allows for divergence in the short run. This is because the expected convergence path for these type of models depends not only on the convergence parameter, $\pi$, but also on the direction of the convergence component at the end of the sample ${ }^{11}$.

More generally, both the general properties of the model and the results presented here, place the 'speed of convergence debate', prevalent in most empirical studies of convergence, in a different perspective. If second-order convergence components best characterise the series, no single parameter captures the speed of convergence and a simple notion of a half-life is inappropriate.

Finally we contrast the behaviour of the two richest regions with that of the six poorest. To this end we fitted a bivariate model, (6), without a convergence component, to NE and ME and extracted the trends. Figure 11 shows the differences between each of these trends and the common trend for the six remaining regions. The divergence is clear.

\footnotetext{
${ }^{11}$ This point is in part related to the call by Durlauf (2001:68) for 'the development of varying coefficient methods that allow the growth process to depend on a country's state of development'.
} 


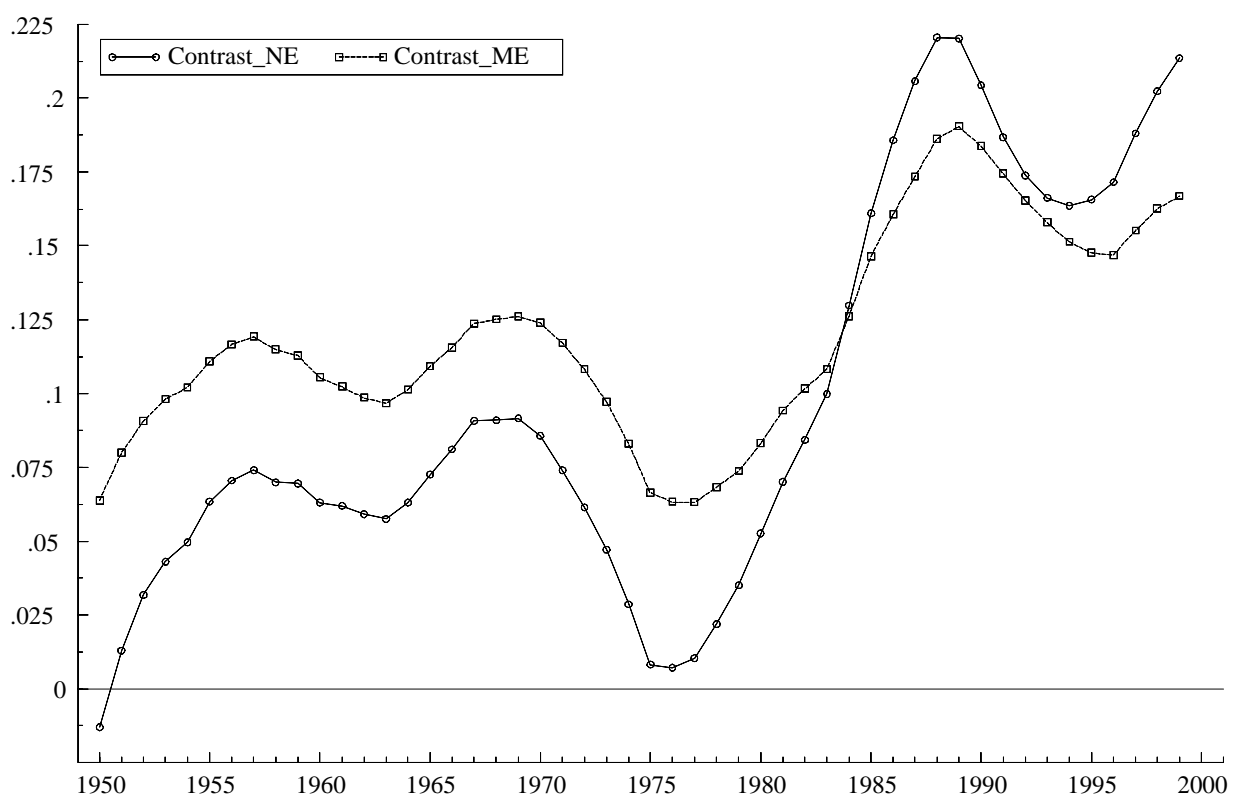

Figure 11: Contrast of two wealthiest regions' trends with common trend of the six poorest regions.

Figure 11 above depicts the difference between the smooth trends of the two wealthiest regions and the estimated balanced growth path of the remaining regions. Comparing to Figure 10, start by noticing that in the early fifties the ME and NE contrasts were actually of a smaller magnitude than that of FW and not very different from that of GL. However, as Figure 12 makes clear, instead of the converging dynamics of the latter two regions, NE and ME actually widen their gap towards other regions, especially from the mid seventies onwards. Thus, it is not the initial state but actually the distinct growth dynamics of NE and ME during the last half century that explain the divergence already hinted at in Section 4.

\section{Testing Convergence}

The aim of convergence tests is to determine whether regions are in the process of converging. Some unit root tests are sensitive to initial conditions, rendering them of 
limited value for this purpose. Harvey and Bates (2002) examine a number of tests and conclude that the ADF $t$-test is the most satisfactory one as it is robust to initial values different from zero. Indeed, when there is no constant, its power increases the further the initial conditions are from equilibrium. Note that many researchers seem to be concerned with testing whether convergence has actually taken place. For example, Bernard and Durlauf (1996 p 171) write that 'In time series tests, one assumes that the data are generated by economies near their limiting distributions and convergence is interpreted to mean that initial conditions have no (statistically significant) effect on the expected value of output differences.' If this really were the case then stability tests, rather than unit root tests, would be appropriate.

The Monte Carlo experiments in Harvey and Bates (2002) also highlight the considerable advantages of tests without the constant when absolute convergence is the hypothesis of interest. Many studies, for example Carlino and Mills (1993), carry out ADF $t$-tests with a time trend as well as a constant. However, the inclusion of a time trend is not only inconsistent with a convergence model but it also effectively ensures that the tests have virtually no power.

The usual approach to testing convergence is based on fitting models of the form ${ }^{12}$ $\Delta\left(y_{i, t}-\bar{y}_{w, t}\right)=\delta_{i}+\pi_{i}\left(y_{i, t-1}-\bar{y}_{w, t-1}\right)+\sum_{r=1}^{p} \phi_{i r} \Delta\left(y_{i, t-1}-\bar{y}_{w, t-1}\right)+\eta_{i t}, \quad i=1, \ldots, N$,

and carrying out ADF unit root tests. In the present application, case $\bar{y}_{w, t}$ would usually be the US figure, rather than a simple weighted of the individual figures in logarithms.

An overall test of convergence is often carried out by combining the information in the individual ADF statistics as in ${ }^{13}$ Evans and Karras (1996a) or Levin, Lin and $\mathrm{Lu}$ (2002). However, these tests do not take account of the cross-correlation between the series, thereby rendering them invalid in the present application; see the simulation evidence in O'Connell (1998). More generally there is the issue of how useful an overall test is in the first place. As we have seen for the US, some regions may converge while others do not. Thus individual tests may be more informative. Unfortunately basing such tests on the equations in (32) in an attempt to determine

\footnotetext{
${ }^{12}$ This equation does not follow from the model of section 5 except in the homogenous case when all the $\pi_{i}^{\prime} s$ are the same; see subsection 5.2 .

${ }^{13}$ The fact that the information in one of the equations is redundant is rarely mentioned.
} 
which regions converge to the overall mean is obviously invalid since if one region does not converge but all the others do, then $y_{i, t}-\bar{y}_{w, t}$ will be nonstationary for all $N$ and the tests tell us nothing ${ }^{14}$. Our preference is therefore to use pairwise ADF tests $^{15}$ to try to determine which regions are converging to each other.

Tables 2 and 3 show the results of the ADF tests, with $p$ set to four, applied to the differences between all the (logged) annual series. The results for the test with no constant show some support for convergence within the six poorest regions, though only six out of fifteen of the test statistics are statistically significant at the $5 \%$ level. As expected, there is no evidence for convergence of $N E$ and $M E$ with any of the other regions or with each other.

If a constant is included, only one $t$-statistic is statistically significant at the $10 \%$ level and this is between $M E$ and $R M$ ! Table $3 \mathrm{~b}$ shows estimates of the constant and associated $t$-tests, though given the complete ineffectiveness of the unit root tests, this may not contain much information of value.

Overall the tests are not inconsistent with the conclusions obtained by the presentation of stylised facts from our models. However, relying solely on the tests would clearly not be a sensible strategy.

\footnotetext{
${ }^{14}$ Nevertheless a vast number of papers report the results of this fruitless exercise.

${ }^{15}$ Hobijn and Franses (2000) propose using pairwise stationarity tests, but as already argued stationarity tests are of little value if the regions are in the process of converging.
} 
Table 2 Annual Data ADF $\tau$ test with 4 lags and no constant

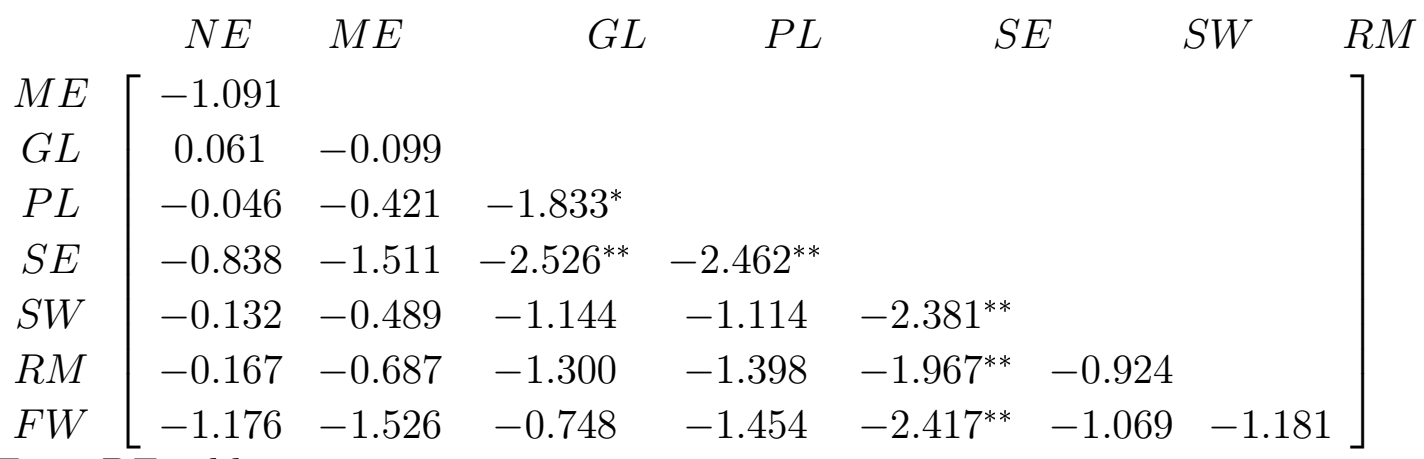

From DF tables:

* means significant at $10 \%$ level (50 observations), critical value $=-1.61$

** means significant at $5 \%$ level (50 observations), critical value $=-1.95$

Table 3a Annual Data ADF $\tau$ test with 4 lags and constant

\begin{tabular}{|c|c|c|c|c|c|c|c|}
\hline & $N E$ & $M E$ & $G$ & & $P L$ & & $W$ \\
\hline$M E$ & -0.381 & & & & & & \\
\hline$G L$ & -0.757 & -1.341 & & & & & \\
\hline$P L$ & -1.681 & -1.792 & -1.361 & & & & \\
\hline$S E$ & -1.796 & -1.785 & -1.876 & -1.819 & & & \\
\hline$S W$ & -2.169 & -2.356 & -1.258 & -2.439 & -2.265 & & \\
\hline$R M$ & -1.689 & $-2.753^{*}$ & -1.850 & -1.653 & -1.446 & -1.195 & \\
\hline$F W$ & -0.685 & -1.299 & -2.110 & -0.416 & -1.213 & -0.954 & -1.112 \\
\hline
\end{tabular}

From DF tables:

${ }^{*}$ means significant at $10 \%$ (50 observations), critical value $=-2.60$

** means significant at 5\% (50 observations), critical value $=-2.93$

Table 3b Annual Data - estimate of constant and associated t-test

$\begin{array}{llllll}N E & \text { ME } & P L & S E & S W & R M\end{array}$




\begin{tabular}{|c|c|c|c|c|c|c|c|}
\hline$M E$ & 0.0012 & & & & & & \\
\hline$G L$ & 0.0039 & 0.0072 & & & & & \\
\hline$P L$ & $0.0216^{*}$ & $0.0294^{*}$ & 0.0032 & & & & \\
\hline$S E$ & 0.0123 & 0.0077 & 0.0009 & 0.0032 & & & \\
\hline$S W$ & $0.0219^{* *}$ & $0.0228^{* *}$ & 0.0059 & $0.0156^{* *}$ & -0.0029 & & \\
\hline$R M$ & $0.0118^{*}$ & $0.0268^{* *}$ & 0.0122 & 0.0028 & -0.0005 & -0.0027 & \\
\hline$F W$ & 0.0013 & 0.0004 & $-0.0173^{* *}$ & -0.0004 & -0.0005 & -0.0059 & -0.0121 \\
\hline
\end{tabular}

\section{Conclusions}

Fitting a multivariate structural time series model to the eight US regions provides considerable insight into convergence, or lack of it, by focussing attention on the extraction of smooth trends. Based on these trends, a plausible working hypothesis is that all but the two richest regions, $\mathrm{NE}$ and $\mathrm{ME}$, have displayed absolute convergence over the last fifty years. A convergence model is developed and fitted to the six poorest regions. A key feature of the model is that it embodies convergence components that are able to display temporary divergence before converging to a common trend. This temporary divergence seems to be a feature of the US regions. The model not only characterises it over the period in question, but also displays it when predictions are made.

Unit root tests are applied to all pairs of regions, it having been noted that models based on deviations from an overall mean are inappropriate when at least some regions diverge. The results of these tests are not inconsistent with the conclusions reached by our presentation of stylised facts. However, in themselves they are inconclusive. Furthermore, they are based on a first-order model of convergence, while our preference is for eliciting stylised facts is a second-order model. Our view is that it is unwise to attempt to draw definitive conclusions on the basis of such tests.

A secondary aim of the study is to characterise the cyclical movements in US regions. We do this by fitting a similar cycle component and the result is individual regional cycles that appear entirely plausible in the light of the national cycle as dated by the NBER. The first principal component of the estimated covariance matrix of the cycles accounts for the bulk of the regional movements and tracks the national cycle quite closely. However, attempts to impose a single common cycle on the regional series indicated that this is too strong an assumption. 
Returning to convergence, there are sound economic reasons why regional per capita incomes should tend to equalise. For example, in the case of the US, the recent paper by Caselli and Coleman (2001) highlights the declining role of agriculture in bringing about convergence. Explaining the underlying reasons for the recently observed divergence of the $\mathrm{NE}$ and $\mathrm{ME}$ presents an even more interesting challenge.

\section{ACKNOWLEDGEMENTS}

The work was supported by the Economic and Social Research Council (ESRC) as part of a project on Dynamic Factor Analysis, grant number L138 25 1008. An earlier version of the paper was presented at the conference on business cycles at the University of Manchester in June 2002.

\section{References}

[1] Barro, R.J. and X. Sala-i-Martin (1992). Convergence. Journal of Political Economy, 100, 223-251.

[2] Barro, R.J. and X. Sala-i-Martin (1995). Economic Growth. McGraw-Hill, Boston, MA.

[3] Bernard, A. and S. Durlauf (1995). Convergence in international output. Journal of Applied Econometrics, 10, 97-108.

[4] Bernard, A. and S. Durlauf (1996). Interpreting tests of the convergence hypothesis. Journal of Econometrics, 71, 161-173.

[5] Brock, W. and S. Durlauf (2000). Growth economics and reality. NBER Working Paper, n. 8041.

[6] Brown,, S.J., N.E. Coulson and R.F. Engle (1990). Non-cointegration and econometric evaluation of models of regional shift and share. NBER Working Paper, n. 3291.

[7] Carlino, G. and L. Mills (1993). Are U.S. regional incomes converging? A time series analysis. Journal of Monetary Economics, 32, 335-346. 
[8] Carlino, G. and K. Sill (2001). Regional Income Fluctuations: Common Trends and Common Cycles. Review of Economics and Statistics, 83.

[9] Caselli, F. and W.J.Coleman (2001). The U.S. structural transformation and regional convergence: a re-interpretation. Journal of Political Economy, 109, 584-616.

[10] Doornik, J. A. (1999). Ox: An Object-Oriented Matrix Language. 3rd edition. Timberlake Consultants Press: London.

[11] Durlauf, S. (2001). Manifesto for a growth econometrics. Journal of Econometrics, 100, 65-69.

[12] Durlauf, S. and D. Quah (1999). The new empirics of economic growth. In J.B. Taylor and M. Woodford (eds.). Handbook of Macroeconomics. Volume 1, Chapter 4. Elsevier Science.

[13] Evans, P. and G. Karras (1996a). Convergence revisited. Journal of Monetary Economics, 37, 249-265.

[14] Evans, P. and G. Karras (1996b). Do economies converge? Evidence from a panel of U.S. states. Review of Economics and Statistics, 78, 384-388.

[15] Evans, P. (2000). Income dynamics in regions and countries. In G. Hess and E. van Wincoop (eds.). Intranational Macroeconomics, Chapter 7. Cambridge University Press: Cambridge.

[16] Engle, R.F. and S. Kozicki (1993), Testing for common features, Journal of Business \& Economics Statistics, 11, 369-380.

[17] Hamilton, J.D. (1994), Time series analysis, Princeton, Princeton University Press.

[18] Harvey, A.C. (1989), Forecasting, Structural Time Series Models and the Kalman Filter. Cambridge: Cambridge University Press.

[19] Harvey, A.C. and D. Bates (2002), Testing for Convergence. Mimeo, Cambridge University. 
[20] Harvey, A.C. and V.M. Carvalho (2002), Models for converging economies. mimeo. DAE Working paper 0216, University of Cambridge.

[21] Harvey, A.C. and S.J. Koopman (1997). Multivariate structural time series models. In: C. Heij et al (eds.). System dynamics in economic and financial models. 269-298. Wiley and Sons: Chichester.

[22] Hobijn, B. and P.H. Franses (2000). Asymptotically perfect and relative convergence of productivity. Journal of Applied Econometrics, 15, 59-81.

[23] Koopman, S.J., N. Shephard and J.A. Doornik (1999). Statistical algorithms for models in state space form using SsfPack 2.2 (with discussion), Econometrics Journal, 2, 107-160.

[24] Levin, A. Lin, C-F. and C.-S. Lu (2002). Unit root tests in panel data: asymptotic and finte sample properties. Journal of Econometrics, 108, 1-24.

[25] Loewy, M.B. and D. H. Papell (1996). Are U.S.regional incomes converging? Some further evidence. Journal of Monetary Economics, 38, 587-598.

[26] Maddala, G.S. and I-M. Kim (1998). Unit roots, co-integration, and structural change. Cambridge: Cambridge University Press.

[27] Nyblom, J. and A.C. Harvey (2000). Tests of common stochastic trends. Econometric Theory, 16, 176-199.

[28] O'Connell, P. (1998), The overvaluation of purchasing power parity, Journal of International Economics, 44, 1-19.

[29] Quah, D. (1993). Galton's fallacy and the convergence hypothesis. Scandinavian Journal of Economics, 95, 427-443.

[30] Sala-i-Martin, X. (1996). Regional cohesion: evidence and theories of regional growth and convergence. European Economic Review, 40, 1325-52.

[31] Tomljanovich, M. and T.J.Vogelsang (2001). Are U.S. regions converging? Using new econometric methods to examine old issues Empirical Economics, 27, 49-62.

[32] Vahid, F. and R. F. Engle (1993). Common trends and common cycles. Journal of Applied Econometrics, 8, 341-360. 


\section{A Appendix}

\section{A.1 Quarterly results for multivariate smooth trend model}

The period for the similar cycle component is 17 quarters with a damping factor of 0.85. Log-Likelihood $=2676.45$. The cycle, trend (slope) and irregular disturbance covariance matrices, are respectively

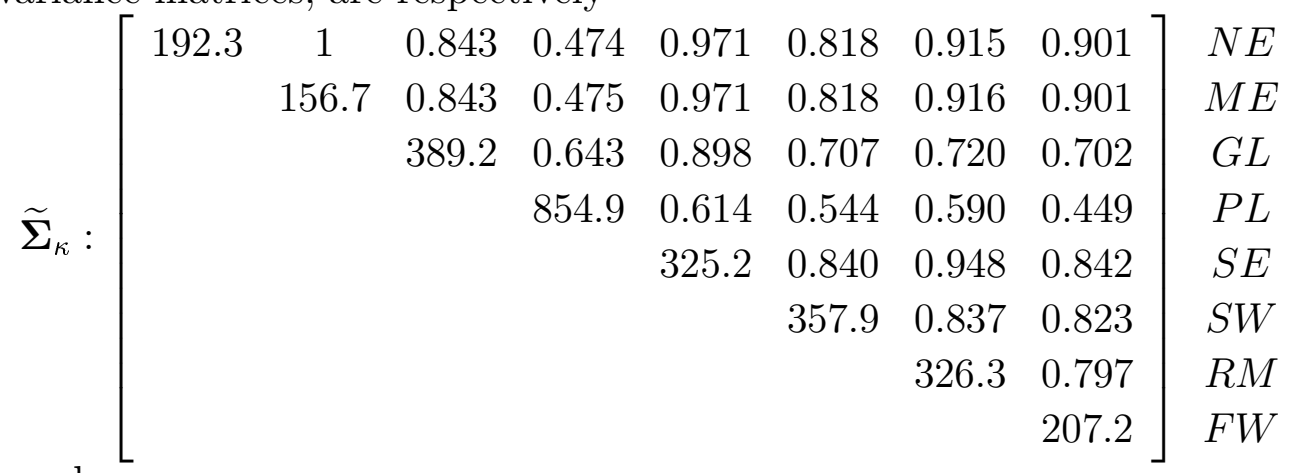

and

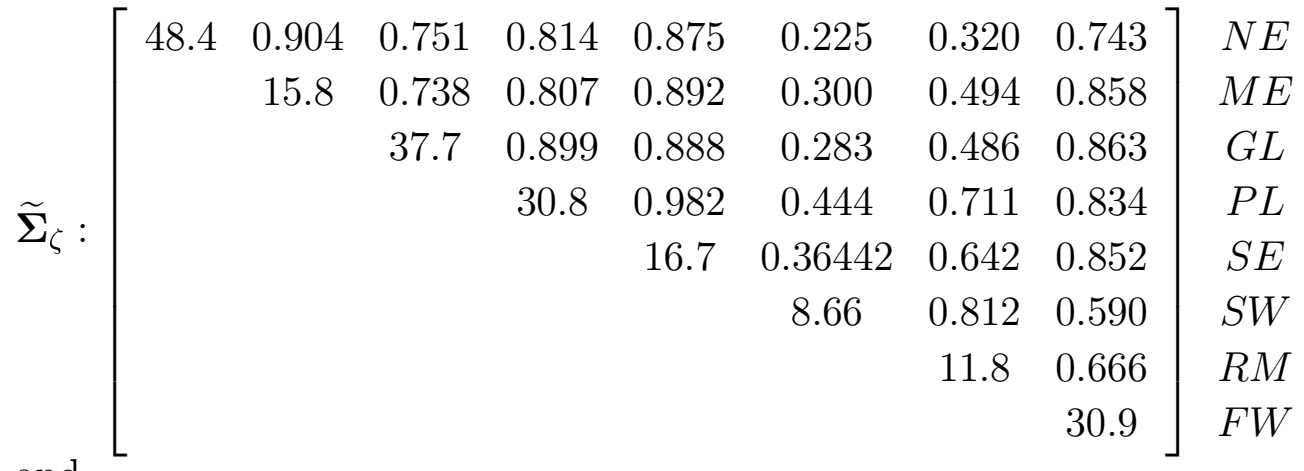

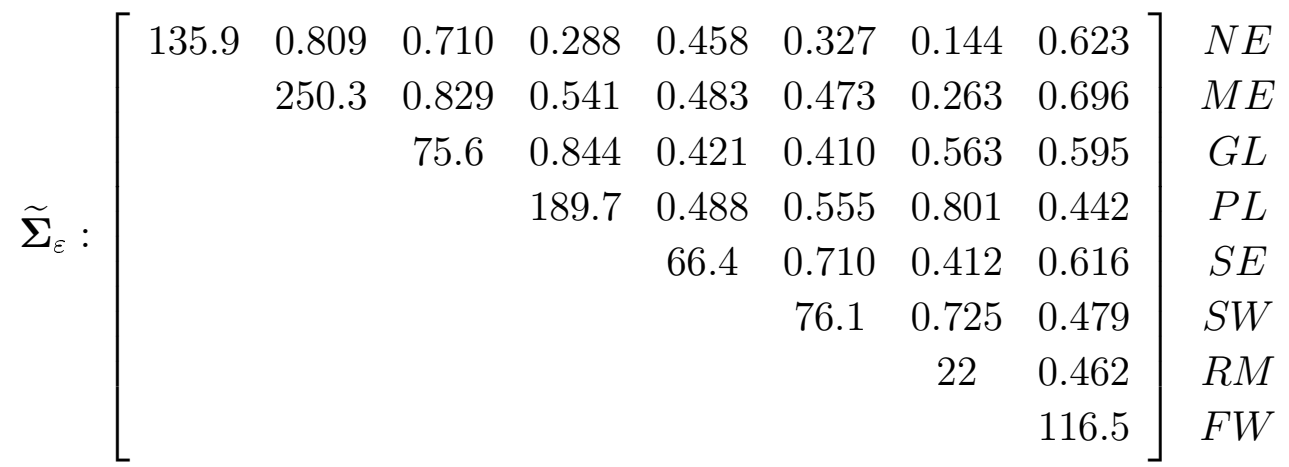


The cyclical and trend components for the eight regional quarterly series are depicted in figures below.

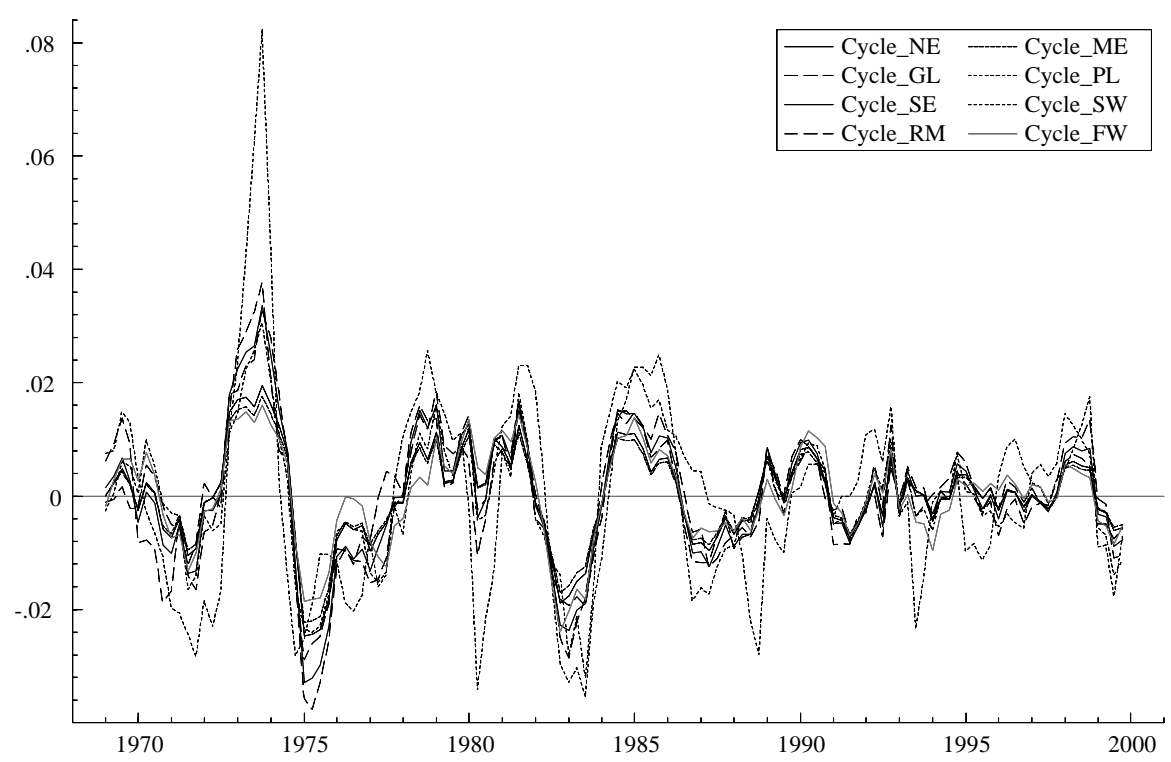

Figure : Smoothed cycles for quarterly data.

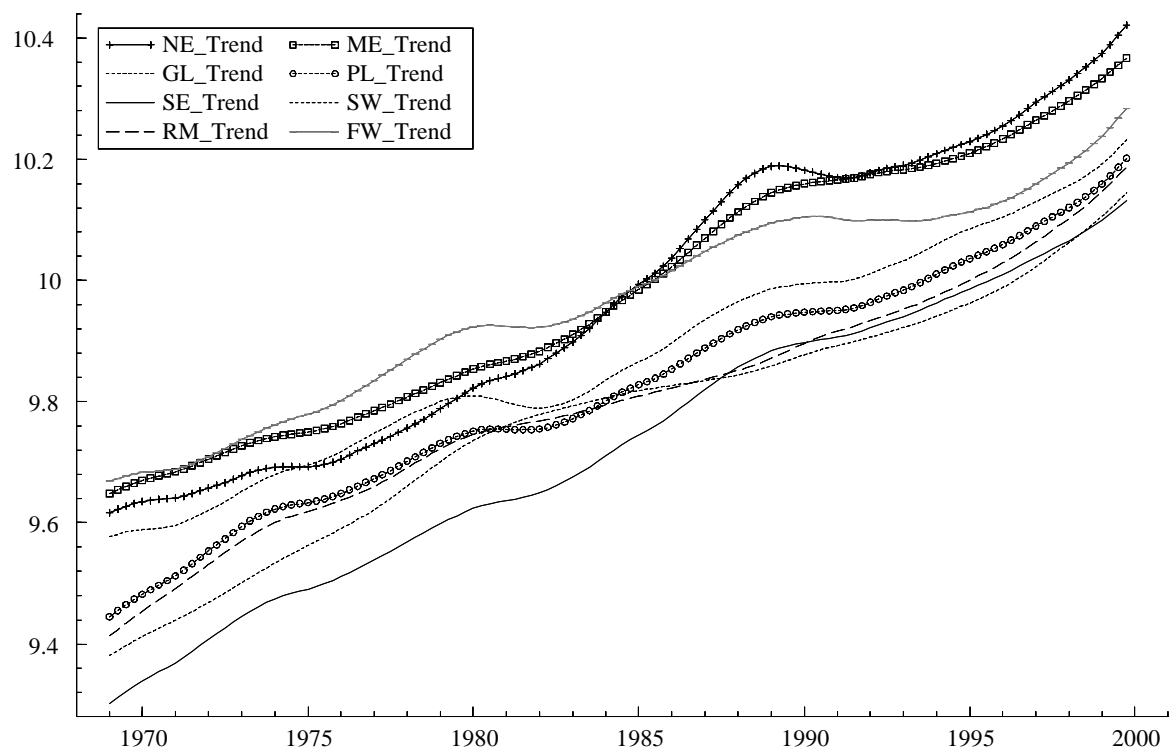

Figure : Smoothed trends for quarterly data. 


\section{A.2 Convergence model for quarterly data (1969:1 to 1999:4) from US six census regions}

Log-Likelihood $=2676.45$. Cycle parameters: $\rho=0.795 ; 2 \pi / \lambda=20$ quarters with covariance matrix (all variances premultiplied by $10^{\wedge} 7$ )

$$
\boldsymbol{\Sigma}_{\kappa}:\left[\begin{array}{cccccc}
311.7 & 0.552 & 0.834 & 0.737 & 0.574 & 0.636 \\
& 857.2 & 0.563 & 0.556 & 0.543 & 0.355 \\
& & 289.1 & 0.882 & 0.927 & 0.808 \\
& & & 433.0 & 0.838 & 0.800 \\
& & & & 315.6 & 0.753 \\
& & & & & 181.7
\end{array}\right] \text { FW }
$$

and for the irregular

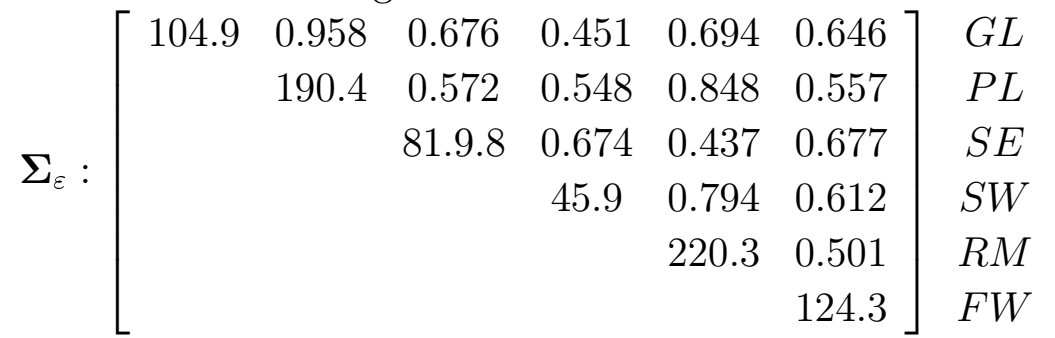

The common trend weights and convergence parameter are:

\begin{tabular}{|c|c|c|c|c|c|c|}
\hline $\bar{\phi}_{G L}$ & $\bar{\phi}_{P L}$ & $\bar{\phi}_{S E}$ & $\bar{\phi}_{S W}$ & $\bar{\phi}_{R M}$ & $\bar{\phi}_{F W}$ & $\pi$ \\
\hline 0.572 & 0.015 & 0.205 & 0.034 & 0.094 & 0.080 & -0.0355 \\
\hline
\end{tabular}

$\boldsymbol{\Sigma}_{\zeta}:\left[\begin{array}{cccccc}86.3 & 0.972 & 0.952 & 0.580 & 0.838 & 0.878 \\ & 79.2 & 0.987 & 0.676 & 0.917 & 0.856 \\ & & 45.8 & 0.584 & 0.865 & 0.826 \\ & & & 16.7 & 0.872 & 0.688 \\ & & & & 31.9 & 0.793 \\ & & & & & 52.9\end{array}\right]$

The graphs are: 


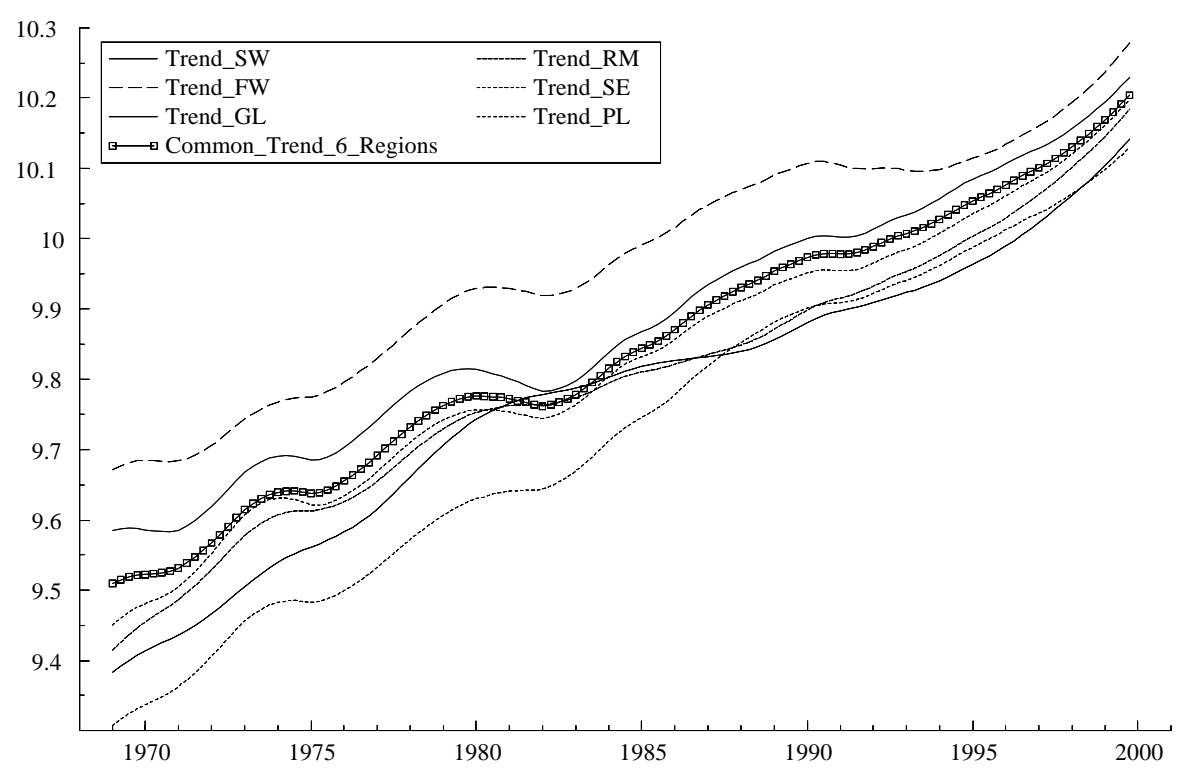

Figure: Smoothed Trend and Common Trend for 6 quarterly series.

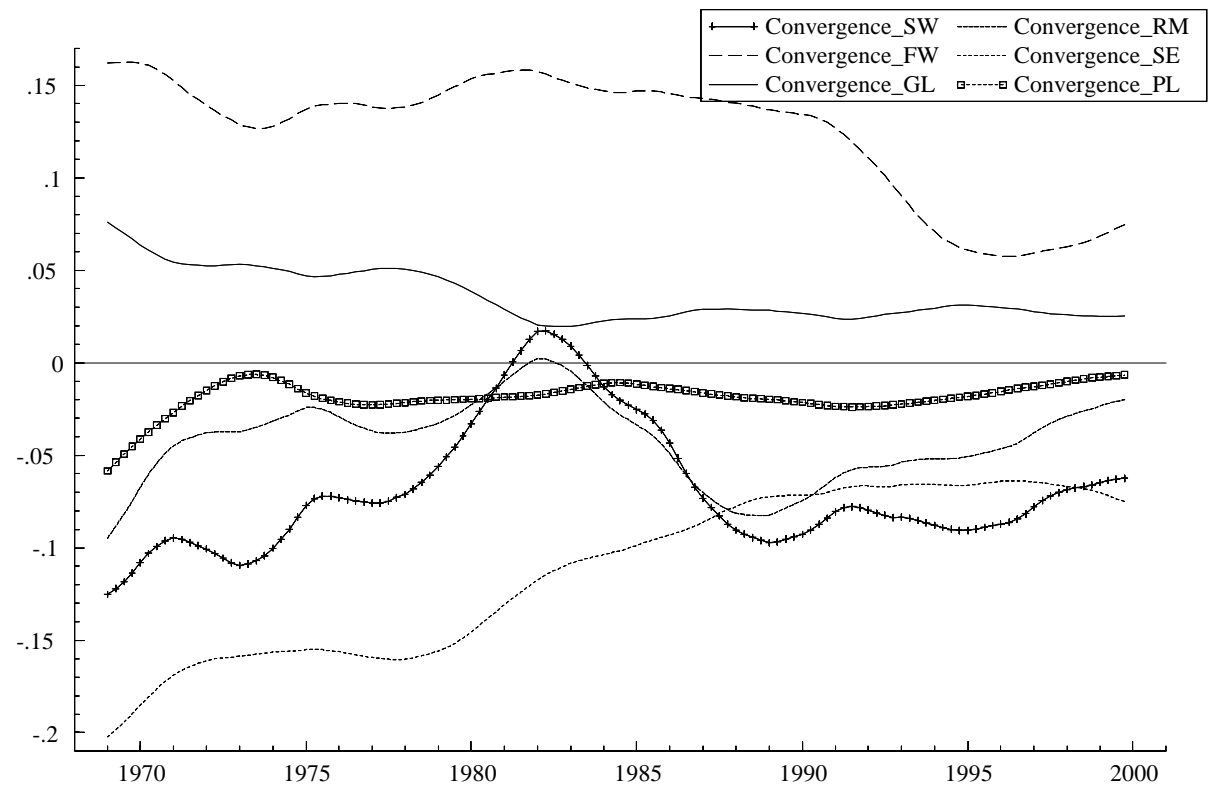

Figure :Smoothed convergence component for 6 quarterly series. 


\section{B Appendix}

\section{B.1 Unit root tests}

Quarterly data, 4 lags, no constant, $\tau$ test

\begin{tabular}{|c|c|c|c|c|c|c|c|}
\hline & $N E$ & $M E$ & $G I$ & & $P L$ & $S E$ & $S W$ \\
\hline$M E$ & -0.633 & & & & & & \\
\hline$G L$ & 0.234 & -0.110 & & & & & \\
\hline$P L$ & 0.113 & -0.502 & $-1.791^{*}$ & & & & \\
\hline$S E$ & -0.135 & $-1.650^{*}$ & $-2.439^{* *}$ & $-1.630^{*}$ & & & \\
\hline$S W$ & -0.204 & -0.546 & -1.111 & -1.092 & -1.478 & & \\
\hline$R M$ & -0.220 & -0.608 & -1.376 & -1.447 & -1.242 & -0.847 & \\
\hline$F W$ & -0.773 & -0.744 & -0.919 & -1.402 & $-1.980^{* *}$ & -1.188 & -1.284 \\
\hline
\end{tabular}

From DF tables:

* means significant at 10\% (100 observations)

** means significant at 5\% (100 observations)

4 lags, with constant, $\tau$ test and constant

A- $\tau$ test

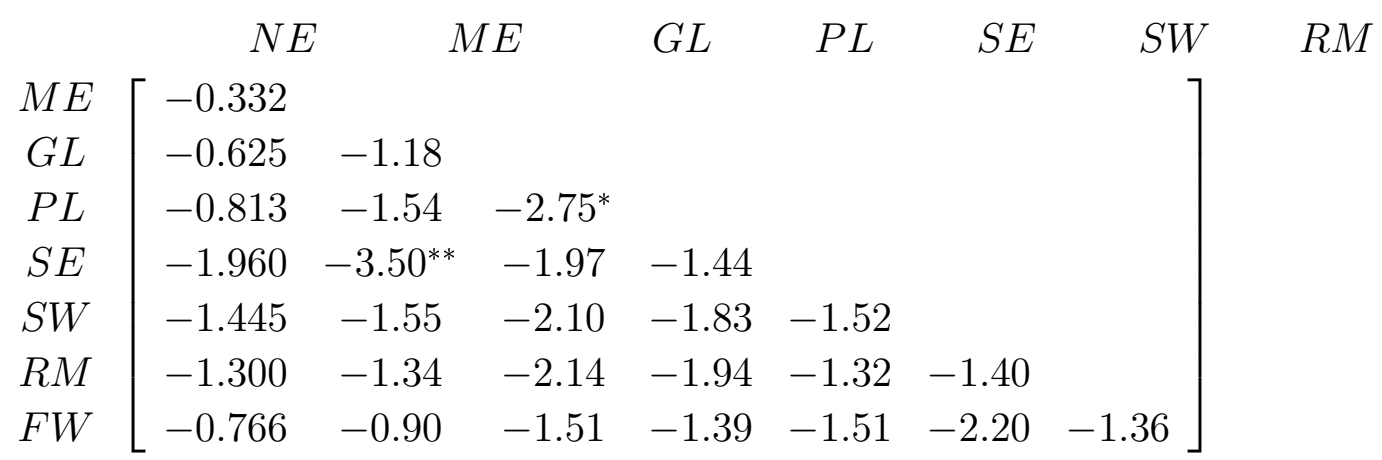

From DF tables:

* means significant at 10\% (100 observations)

** means significant at 5\% (100 observations)

B- Value of Constant 


\begin{tabular}{|c|c|c|c|c|c|c|c|}
\hline & $N E$ & $M E$ & & $G L$ & $P L$ & $S E$ & $S W$ \\
\hline$M E$ & 0.0005 & & & & & & \\
\hline$G L$ & 0.0009 & 0.002 & & & & & \\
\hline$P L$ & 0.003 & 0.007 & $0.005^{* *}$ & & & & \\
\hline$S E$ & $0.007^{* *}$ & $0.02^{* *}$ & 0.0018 & 0.0020 & & & \\
\hline$S W$ & 0.003 & 0.004 & $0.0035^{*}$ & 0.0028 & -0.0004 & & \\
\hline$R M$ & 0003 & 0.004 & $0.0043^{*}$ & 0.0018 & -0.0007 & -0.0010 & \\
\hline$F W$ & 0.0005 & 0.0005 & -0.0024 & -0.0047 & -0.0018 & $-0.0064^{* *}$ & -0.0037 \\
\hline
\end{tabular}

